\title{
Computer Analysis of Cardiac Radionuclide Data
}

\author{
Jerry W. Froelich, James H. Thrall, Victor Kalff, W. Leslie Rogers, and Mark Rabinovitch
}

$\mathbf{T}$ HE impact of computers on Nuclear Cardiology and the progress which has been made through computer applications is perhaps best illustratcd by several historical observations. First, it is of note that all clinically important types of nuclear cardiologic procedures were initially performed without the aid of a computer. For example, the first gated equilibrium, blood pool ventriculograms performed by Zaret et al. ${ }^{1,2}$ were accomplished by analog imaging. A physiologic trigger or gate was used to turn the gamma camera image recording system on and off to define "end systole" and "end diastole" which were the only two images obtained. The gating "window" used for end diastole was the $60-\mathrm{msec}$ interval immediately prior to the Rwave. For end systole, data were recorded during the T-wave. Quantitative analysis was then accomplished manually. The analog images were projected to life size and the ventricular borders traced by hand. Ventricular volumes and ejection fractions were calculated using area length formulae adapted from contrast angiography. The data obtained in this fashion correlated well with results from contrast angiography and provided a strong motivation for further technical development. Likewise, in early applications of the first pass technique, computers were not used. Rather, data were recorded on video tape for subsequent replay and analysis. ${ }^{3}$ These analog imaging techniques were extremely time consuming, particularly for purposes of quantitative analysis, took advantage of only a small fraction of the potentially available data, and provided limited visual appreciation of cardiac dynamics.

The first logical adaptation of the computer to radionuclide ventriculography was simply to acquire the gated end diastolic and end systolic frames into computer memory, instead of onto film, one frame at a time. Initially the data analysis was again performed by hand by tracing ventricular contours on the computer screen. As before, this analytic approach was quite tedious and partly for this reason very little clinical use was made of radionuclide ventriculography, initially. However, several breakthroughs in computer techniques occurred in the early 1970s which established radionuclide ventriculography as a feasible clinical procedure.

First, Secker-Walker and Parker ${ }^{4,5}$ recognized that after equilibration in the blood pool the net ventricular count rate resulting from an intravascular tracer (e.g., Tc-99m albumin or Tc-99m $\mathrm{RBC}$ ) at any time in the cardiac cycle is proportional to ventricular blood volume. This germinal observation permits calculation of the ventricular ejection fraction and other quantitative parameters through analysis of net counts (i.e., background corrected) in the ventricles (a task for which the computer is uniquely suited) rather than by the more laborious geometric technique. The formula for the count based ejection fraction is:

Ejection fraction $=$

\begin{tabular}{l} 
End Diastolic Counts (net) \\
- End Systolic Counts (net) \\
\hline End Diastolic Counts (net)
\end{tabular}

All commercially available Nuclear Medicine computer systems now have automated or semiautomated programs for making this fundamental calculation. The computer-aided calculations are faster, more reproducible and more accurate than hand analyses. ${ }^{6}$ For equilibrium blood pool studies the count based quantitative analysis is usually performed in the left anterior oblique view to provide maximal separation of the left ventricle from scintillations arising in the other cardiac chambers. This is a critical point for equilibrium blood pool studies because of the overlap of the left and right ventricles in the right anterior oblique view which is ordinarily used for geometric analysis. For first pass studies positioning choice is more flexible since the activity in the two ventricles is temporally separated.

Two other important nuclear cardiologic com-

From the Division of Nuclear Medicine. The University of Michigan Medical Center, Ann Arbor, Mich.

Reprint requests should be addressed to Jerry W. Froelich, M.D., Nuclear Medicine and Nuclear Magnetic Resonance Imaging, 2799 West Grand Boulevard, Detroit, MI 48202.

(c) 1983 by Grune \& Stratton, Inc. $0033-6620 / 83 / 2601-0004 \$ 02.00 / 0$ 
puter breakthroughs were the development of new computer acquisition techniques for: (1) gated list mode ventriculography; and (2) multigated frame mode (matrix mode, histogram mode) ventriculography. The gated list mode technique was pioneered by Green ${ }^{7}$ and colleagues working at the National Institutes of Health. In this approach, the computer memory is used to store each scintillation event individually as a list or series (hence the names list mode and serial mode) with interspersed time markers and R-wave gating signals. Thus, at the end of data collection, the individual scintillations can be reformatted into a sequence of images spanning the cardiac cycle using the $\mathrm{R}$-wave markers and clock time markers for correctly locating each event in its proper frame. Moreover, the timing of the $R-R$ intervals can be selected retrospectively so that only data collected while the heart is beating at a given desired rate is included in the study. This is critical in studying patients with arrythmias. (See discussion of list mode acquisition.)

The multigated frame mode technique (MUGA) was pioneered by Strauss et al. working at Johns Hopkins University. ${ }^{8}$ In this approach, the computer memory is used quite differently than in list mode. Image matrices, typically $64 \times 64$ pixels or $32 \times 32$ pixels, are designated in computer memory. The $\mathrm{R}$-wave trigger or "gate" initiates data collection at end diastole into the first frame or matrix location in computer memory and all scintillation data occurring within a small "time window," usually on the order of $30-50 \mathrm{msec}$, is placed in it. During the next "time window" or frame, acquisition is into the second matrix location and so on throughout the cardiac cycle until the next Rwave synchronization signal is received by the computer. At this point, data collection is reinitiated into the first matrix with data being added on top of information already present (Fig. 1). This process is continued through many cardiac cycles until each data frame has a sufficiently high count for statistically valid quantitative analysis and good image quality. The multigated frame mode technique is the most frequently used procedure for radionuclide ventriculography in current clinical practice.

Most Nuclear Medicine computer systems were not capable of performing either the gated list mode or multigated frame mode type of data acquisition when these techniques were first described. The typical Nuclear Medicine computer of the early 1970s consisted of $12 \mathrm{k}$ core memory and was "fast" enough to allow acquisi-

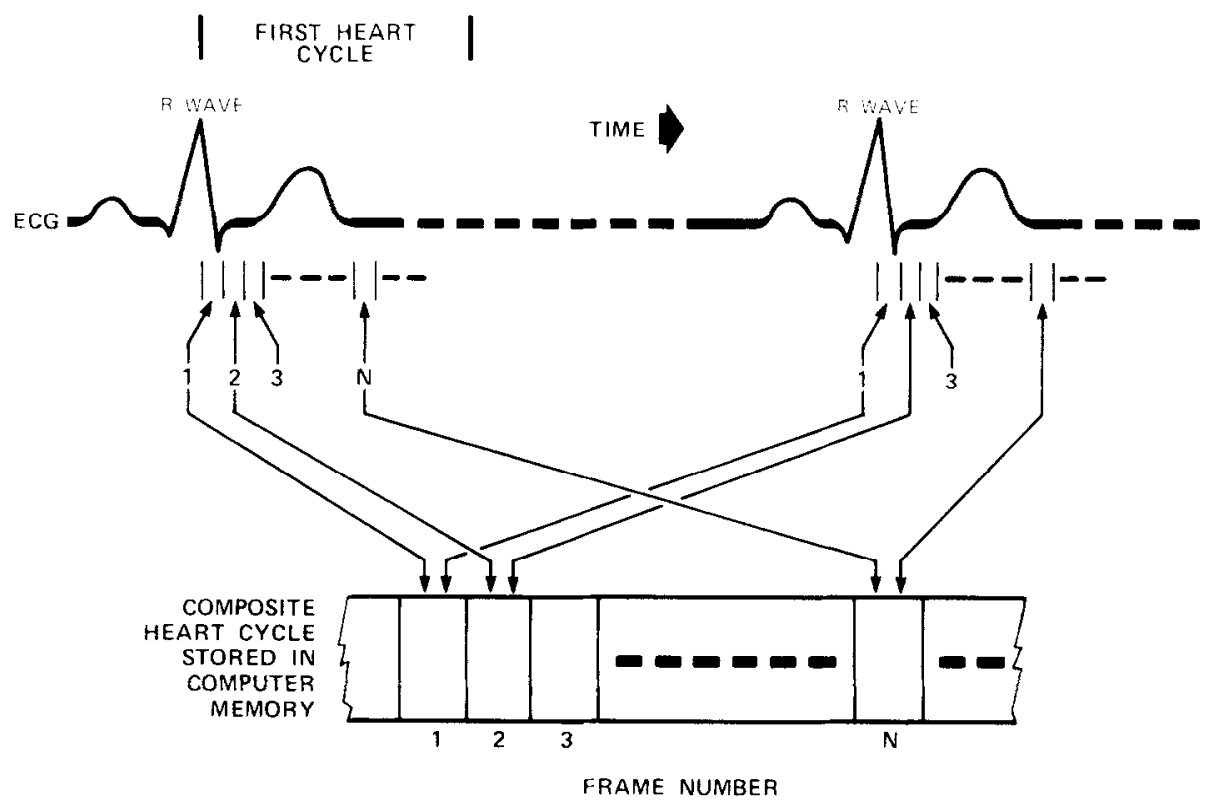

Fig. 1. Schematic diagram of ECG synchronized acquisition. Data collection is initiated when the R wave timing spike is received by the computer. Date from each sequential time segment over the cardiac cycle is stored in computer memory in a predefined matrix or frame buffer. 
tion of dynamic studies (radionuclide flow studies or radionuclide angiograms) at frame rates up to 9 or $10 / \mathrm{sec}$, which is inadequate for quantitative cardiac function analysis. Typically in these early systems a $64 \times 64$ frame $(4 \mathrm{k})$ would be acquired into one $4 \mathrm{k}$ matrix location in memory while the image data in a second $4 \mathrm{k}$ matrix was being transferred to disk or magnetic tape. Part of the remaining $4 \mathrm{k}$ was used for system programs. With the advent of radionuclide ventriculography, computer specifications changed dramatically. The first systems designed specifically for Nuclear Cardiology were configured with $32 \mathrm{k}$ of memory which permitted the acquisition of a typical multigated radionuclide ventriculogram consisting of $28(32$ pixel $\times 32$ pixel $) 1 \mathrm{k}$ frames without the need to transfer data to disk or tape storage during acquisition. It is not unusual for current Nuclear Cardiology systems to have as much as 128 or even $256 \mathrm{k}$ of memory which allows simultaneous acquisition and processing of scintigraphic data from several devices.

Beyond the obvious impact on the design specifications of dedicated Nuclear Medicine computer systems, Nuclear Cardiology has also had a profound impact on overall computer capacity requirements in Nuclear Medicine Departments. For example, in 1968, the Division of Nuclear Medicine at the University of Michigan had a single computer system programmed by paper tape and capable of acquiring a single static image at a time. In 1972, this initial computer was replaced by a $32 \mathrm{k}$ system capable of acquiring static images or slow dynamic studies simultaneously from two different gamma cameras while processing a third study. However, no nuclear cardiologic studies were performed on this computer system until 1976 and it remained the sole computer system in the Division until 1977. In the ensuing five years, an additional seven computer systems have been acquired and currently $60 \%$ of the total computer utilization is related to cardiologic studies. In addition to radionuclide ventriculograms, studies for detection of left to right and right to left shunts, infarct avid scintigrams and thallium myocardial perfusion scans are acquired with the computer. For the infarct avid scans and thallium myocardial perfusion scans Nuclear tomographic imaging has become important. The tomographic techniques require sophisticated computer acquisition and reconstruction programs. Further computer processing of thallium scans for quantitative regional uptake analysis is performed on both planar and tomographic images. Taken together, cardiologic procedures now account for a total of over 150,000 image frames per year acquired by computer in the Division.

\section{EQUIPMENT}

A comprehensive understanding of electronics and computer technology is not necessary for a clinician to perform clinical and research studies on nuclear medicine computers. (In-depth descriptions of gamma camera and computer technology for Nuclear Cardiology are available in textbook form. ${ }^{9-11}$ ) However, basic computer terminology must be understood, since it pervades the literature and discourse of the computer world. Two of the most commonly used words are "hardware" and "software." Hardware is the physical substance of the computer, while software refers to the instructions or "programs" that are stored within the computer for carrying out particular functions. For conceptualization, one can think of the computer hardware as the physical vocal cords within a person while the software is the thought processes which instruct the vocal cords to perform in a particular manner to create speech. The physical components of computers used in Nuclear Cardiology are similar to those used in other applications and are schematically illustrated in Fig. 2.

The following is an overview of the equipment necessary for nuclear cardingraphic procedures.

There are two basic types of computers: digital and analog. In analog computers, information is represented by voltage levels and, it is either the shape or the final voltage level, which represents the information generated by the computer. Analog computers are typically designed to solve specific problems at very high speeds but are not readily "programmed" and thus are not flexible enough for general purpose applications. All commercially available Nuclear Cardiology computer systems are "digital" computers and the discussion will be limited to them.

"Digital" refers to computers which use binary coded numbers (Base 2) for internal representation of information. In this system, numbers are represented by series of digits which 


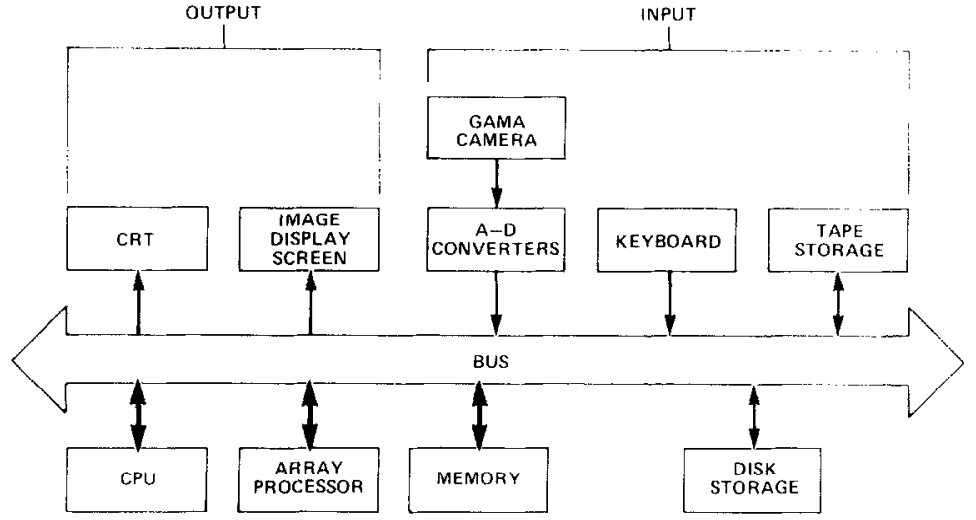

Fig. 2. Schematic diagram of the major components of a digital computer as used in a typical Nuclear Medicing department. have a value of either 0 or 1 . A "bit," or single binary digit, is the smallest piece of information processed by a computer and takes one of these two possible values. This system of representation is ideally suited for electronic computer circuitry where the binary information can be represented cither as a ground or a 5-volt positive voltage.

There are many different subtypes of digital computers marketed for use in nuclear cardiology, including mainframe computers, minicomputers, and microcomputers. At one time, there was a marked degradation in computational speed when one went from the mainframe computer to a microprocessor. The choice of commercial systems was influenced by basic hardware design, however, the rapid advance of computer technology has narrowed the difference between the various types of computers.

Typically, the mainframe computer is a physically large computer which has large quantities of memory and peripheral storage (tape and disk drive) and is capable of performing a much greater number of functions than the mini or microcomputers. The minicomputer can be thought of as a less expensive computer which contains a subset of the functions of the larger mainframe computers. Since the minicomputers contain fewer components, their price is significantly lower.

Over the last few years, the microprocessor, once considered an extremely limited computational device, has been designed with the complexity and the instruction size of larger mainframe computers. In microcomputer technology, the computer is composed of a single integrated circuit performing the functions of a much larger number of conventional components. With its development, the distinction between different subtypes of the digital computer is no longer as clearcut and is less important in selecting a system for clinical use.

There are other hardware considerations that must be kept in mind when one is exploring the purchase of a Nuclear Medicine computer. If an existing gamma camera or a gamma camera from a different manufacturer is to be used with a particular computer, then the interfacing requirements of the camera/computer must be considered. The gamma camera/computer interface is basically an analog to a digital conversion unit which takes the voltage impulses from the gamma camera and converts them to digital numbers that the computer can utilize.

Much emphasis has also been placed on the size of memory within the computer. Typically, memory is measured in terms of thousands of bytes ( 1 Byte $=8$ Bits $)(1 k=1024$ Bytes $)$. Sales people may try to impress the purchaser with the magnitude of memory that can be used within their machine. One must keep in mind that the particular clinical applications will usually dictate the amount of computer memory required. With some machines the increase in memory size will allow more users while the usable memory for any one user is fixed. Memory can be rather costly so the purchase of equipment that will not be utilized should be avoided.

\section{Image Display}

The resolution of images as acquired on the computer is dictated by a matrix or composite of small elements called pixels (picture element). The number of pixels within the matrix (i.e.. 
$32 \times 32,64 \times 64,128 \times 128,256 \times 256)$ dictates the resolution. The higher the number of pixels, the finer the resolution.

Each pixel has a value associated with it that represents the number of counts that can be recorded within that area of the image. To understand the definition of a "pixel," we can use the standard newspaper picture as a conceptual tool. The newspaper picture is composed of a large number of "dots" of different size and packing density to represent bright and dark areas within the picture (image). With computer images in Nuclear Medicine, we can vary the number of dots, the matrix size as measured in pixels, as well as the brightness of each pixel. The depth of a pixel refers to the magnitude of the number that can be stored within that area of the image. Pixel depth actually refers to two separate uses; image display pixel depth and the depth required to store an image. For statistical reasons a certain magnitude of counts must be acquired to truly reflect the distribution of activity, requiring storage of between 256 and 1024 events per pixel. There are three commonly available pixel depths, 256 ( 8 bits), 1024 ( 10 bits), and 65536 (16 bits). For display, the pixel depth is translated into brightness levels. The human eye is capable of perceiving brightness changes of $1 \%$ $2 \%$, thus a video display capable of 64 levels (requiring 6 bits) of intensity would maximally cover the perceptive brightness changes of the eye. Note that these steps in intensity or brightness are not gray levels in the traditional logarithmic sense, but a division of the scale from dark to bright in 64 steps. All current machines should be able to acquire into a $256 \times 256$ matrix and should have a minimum of 8 bits of depth per pixel (able to store 256 events/pixel) and preferably 10 bits or more as an option.

The image matrix needs to be displayed on a computer monitor which is capable of reproducing the information in a manner which does not degrade the image quality. While the black and white monitor is normally standard on each computer, color monitors are favored by some. Some manufacturers offer computers which are able to display information in both color and black and white on two separate monitors at the same time.

Another computer option which is frequently talked about is the "array processor." The purpose of this unit is to speed up arithmetic and array calculations. In a Fourier transform (required for phase analysis), thousands of mathematical computations are necessary and the array processor speeds up the calculations frequently by a factor of 10 or more. The trade-off with the array processors is the cost which ranges from $\$ 10,000-\$ 20,000$ on small systems.

\section{Mass Storage}

Another major piece of hardware required for Nuclear Cardiology computer systems is a peripheral storage device. Peripheral storage is a way of storing studies or data which are rapidly acquired and cannot be held in memory at the time of analysis or acquisition. Older computer systems frequently utilized magnetic tape for primary mass storage while newer systems contain one or more disks. Disk storage is currently measured in terms of megabytes ( 1 megabyte $=$ 1 million bytes). These storage devices range in size from 1 megabyte on a flexible disk to greater than 80 megabytes on larger multiplatter disks. Particular applications, such as list mode acquisition, require a disk storage device to acquire and analyze the data. As was stressed earlier, the specific applications and the size of the clinical workload should dictate the storage requirements of the disk unit.

A description of computers is not completc without a description of software. Companies which market Nuclear Medicine computers are responsible for writing the application software to utilize fully their systems and to comprehensively perform nuclear medicine studies. Computer hardware technology has progressed to the point that the main consideration of a potential purchaser of computer equipment is often the available software. A logical approach to take in choosing appropriate equipment is first to develop an inventory of the studies which will be performed. These can then be matched against the list of available programs provided by the vendor. The user should ask the vendor if the programs have been clinically verified and if the range of normals utilizing their software is available. If the vendors cannot supply this information then the accuracy of the programs must be questioned. 
If on-site development is to be undertaken, there is an added necessity to be able to modify or add software to the existing system. The addition of new programs is usually carried out through a high level computer language, typically FORTRAN. Some manufacturers can also accomplish this through the use of another language called BASIC. The pros and cons of the different languages have been covered in the textbook by Holman and Parker.'

\section{Gamma Camera}

In an article discussing computers in Nuclear Medicine, it may seem inappropriate to discuss gamma cameras, but the camera is the device that limits resolution and defines the site where studies can be performed. One manufacturer has introduced a camera that is dependent on the computer attached to it in order to provide enhanced uniformity and linearity correction. This camera-computer combination allows the acquisition of count rates never before achieved. Because of this growing relationship of camera dependent on computer the authors have included a brief discussion of gamma cameras.

Gamma cameras employ a single, large area, sodium iodide (Thallium Activated) crystal, usually $12^{\prime \prime}$ to $20^{\prime \prime}$ in diameter. The large diameter crystals are used for stationary cameras while the smaller diameter crystals are used for portable cameras.

Each gamma camera contains an array of photomultiplier tubes coupled directly to the crystal or through light pipes. Most modern gamma cameras contain 37 photomultiplier tubes of 2 " diameter arrayed in a hexagonal pattern while other manufacturers employ as many as 91 photomultiplier tubes. For cardiovascular studies, a single peak (the ability to look at a single energy peak) capability is all that is necessary. When isotopes other than technetium or thallium are to be utilized, then multi-energy capabilities should be considered.

The gamma camera should have uniformity correction which is usually specitied for the major portion of the field of $90 \%$ or better. Uniformity correction electronics maintain a uniform image thus compensating for equipment variations. Linearity, the ability to represent straight sources or edges, in the camera display, should not exhibit variances of greater than $1.5 \%$.

For cardiovascular laboratories performing only thallium and technetium studies, a sodium iodide crystal thickness of $1 / 4^{\prime \prime}$ is sufficient. If higher energy isotopes are to be used, then a $1 / x^{\prime \prime}$ or a $1 / 2$ " crystal is necessary in order to maximize the intrinsic photo peak efficiency.

For $140 \mathrm{Kev}$ gamma rays interacting with a $1 / 4^{\prime \prime}$ crystal, the intrinsic photo peak efficiency is $70 \%$ compared to $90 \%$ for a $1 / 2^{\prime \prime}$ thick crystal. The $1 / 4^{\prime \prime}$ crystal would require either a larger dose or longer imaging time to achieve the same statist jcal accuracy as the $1 / 2 "$ crystal. At energies less than $100 \mathrm{Kev}$ (i.e., thallium), a $1 / 4$ " crystal has a photo peak detection efficiency greater than $95 \%$ and a crystal thickness greater than $1 / 4$ " only contributes to a decrease in spatial resolution as at result of scattering of light photons within the crystal. The $3 / s^{\prime \prime}$ crystal has been offered as a compromise between the $1 / 2$ " and the $1 / 4^{\prime \prime}$. Remember, there is always a trade-off between resolution (thinner crystal) and efficiency (improved with thick crystal).

A special type of gamma camera and computer is the "mobile" imaging and acquisition device. This is a special purpose gamma camera and computer which allows the acquisition of studies at a site remote from the main clinical unit. The requirements and options for this device are typically comparable to a subset of the larger computers. A study by Royal et al.'2 contains a comparison of various mobile gamma cameras.

The ability to do studies at the bedside requires a mobile gamma camera and computer. Various manufacturers offer gamma cameras and computers for portable studies with both integrated and external computers. As the number of interventional and bedside studies are increased, there will be a greater need to utilize portable gamma cameras in Nuclear Cardiology.

\section{RADIONUCLIDE VENTRICULOGRAPHY}

As discussed above the logical first step for computers in nuclear cardiology was development of data acquisition techniques for radionuclide ventriculography. The acquisition programs are now fairly well defined and, in fact. 
Table 1. Functional Parameters Measured in Gated Cardiac Blood Pool Scintigraphy

1. Wall motion assessment Regional
2. Ejection fraction
3. End diastolic and end systolic volume Regional
4. End systolic volume/peak systolic pressure
5. Stroke volume and cardiac output
$\begin{aligned} & \text { 6. Regurgitant fraction } \\ & \text { 7. Systolic emptying and } \\ & \text { diastolic filling rate }\end{aligned}$
$\begin{aligned} & \text { 8. Time to peak systolic emptying and diastolic } \\ & \text { filling }\end{aligned}$

have not evolved significantly in the past three or four years. During this time, major efforts have been directed at development of new software programs to permit calculation of several new quantitative parameters in addition to the ejection fraction of the left ventricle and to permit analysis of ventricular function in patients with ccrtain arrythmias. Table 1 summarizes many of the functional parameters now available through the development of new computer software. Although it is beyond the scope of this article to discuss each of these in detail, it is important to recognize which are considered well established and which must await further confirmation through additional clinical evaluation.

Excellent correlations of left ventricular ejection fraction computed from radionuclide ventriculograms and from contrast angiograms have been presented by numerous laboratories throughout the country. ${ }^{6.13-16}$ The measurement is now universally accepted and for several clinical problems, such as ventricular aneurysm, is felt to be more accurate than the ejection fraction calculated from contrast angiograms because no geometric assumptions need be made about the shape of the ventricle.

Measurement of right ventricular ejection fraction is less well established, in part, because of the lack of a completely accepted standard of comparison and, in part, because of certain theoretical limitations on radionuclide studies. In first pass ventriculograms, radioactivity in the right heart is temporally isolated from activity in the left ventricle. The count based approach to computing right ventricular ejection fraction is straightforward and has proven useful clinically. ${ }^{17}$ Incomplete mixing of radiotracer in the right ventricle is a theoretical problem especially if there is significant enlargement. The calculated ejection fraction may be too high with incomplete mixing.

For the more commonly performed equilibrium blood pool approach to ventriculography, it is generally not possible to isolate the right ventricle from both the left ventricle and the right atrium in any single view. Although good correlations of the right ventricular ejection fraction calculated from equilibrium blood pool studies and first pass studies have been reported by several groups, ${ }^{18-20}$ the major theoretical problem of chamber overlap remains and not all investigators have confirmed these results. In addition to chamber overlap, incorrect definition of the pulmonic valve plane is a potential source of error. It is also more difficult to flag regions of interest for the right ventricle due to its irregular shape compared to the left ventricle.

The regional cjection fraction has been proposed as a method for detecting abnormalities in segmental or regional wall motion. ${ }^{21-23}$ Numerous schemes have been proposed for effecting this analysis and have been, in part, derived from angiographic techniques. The ventricle is divided into segments and a count-based ejection fraction computed for each segment. While the concept is very attractive, the major difficulties yet to be overcome are the lack of "normal" limits for regional ejection fractions to distinguish normal from abnormal segments, the difficulty of assigning appropriate background corrections on a regional basis, particularly along the interventricular septum, and the problem of rotational and translational movements of the heart which frustrate any attempts at subdividing the ventricle in a strict geometric way.

Ventricular volume has been measured using both geometric principles and count-based tech- 
niques. ${ }^{2.24-16}$ The geometric approach uses formulae adapted directly from contrast angiography. The two critical steps are: (1) rescaling the image to correct for minification; and (2) definition of the ventricular contour. The rescaling step is straight forward using digital computers because the size of the pixels is readily calibrated. For example, with a standard field-of-view gamma camera (10-in.) and a $64 \times 64$ matrix, each pixel represents roughly a $4 \times 4-\mathrm{mm}$ area. Definition of the ventricular contour is either accomplished by hand drawing with the computer light pen or by using automated edge detection techniques. The geometric approach is difficult for equilibrium blood pool images because of chamber overlap in the right anterior oblique view but has been applied extensively in first pass studies. When the geometric technique is used for computer analysis of radionuclide ventriculograms, the same assumptions must be made as are made for contrast angiography; that is, the ventricle is cllipsoidal at end diastole and maintains a similar shape during the cardiac cycle. This is a clear limitation of the geometric approach in patients with ventricular aneurysms and/or other significant segmental wall motion abnormalities.

More recently, count-based techniques have been described for computing volumes. ${ }^{24.26}$ Again, the underlying principal is that the net ventricular activity at any point in the cardiac cycle is proportional to ventricular blood volume. Therefore, if a small reference blood sample ( 5 $\mathrm{ml}$ ) is obtained by venipuncture at the time of imaging and the counts $/ \mathrm{sec} / \mathrm{ml}$ determined on the gamma camera, the ventricular volume may be computed as the ratio of net ventricular counts to blood counts $/ \mathrm{ml}$.

Ventricular Volume

$($ End Diastole $)=$

Net Ventricular Count/sec (End Diastole)

Blood Counts $/ \mathrm{sec} / \mathrm{ml}$

This would be true if the counting efficiency were the same for both measurements and no attenuation of radioactivity occurred in the chest wall. Unfortunately, neither of these assumptions holds. By far the more important factor is attenuation of activity in the chest wall and several approaches have been described for attentuation correction. For ${ }^{99 \mathrm{~m}} \mathrm{Tc}$ the attenuation loss is approximately $14 \%$ for each centimeter of soft tissue between the detector and the source. Thus, one approach to attenuation correction is to measure the depth of the centroid of ventricular activity and directly compute an attenuation correction factor. ${ }^{26}$ Depth may be determined by placing a marker source of radioactivity on the patient's chest and obtaining a second view with a known geometric relationship to the standard view. The distance from the marker source to the center of the ventricle is then measured in the second image. Ultrasound has also been used to calculate ventricular depth.

A slightly different approach has been described independently by Dehmer ${ }^{25}$ and by Slutsky. ${ }^{24}$ In their approach, an average correction factor for attenuation and counting efficiency is computed through regression analysis of radionuclide and contrast angiographic results in a "lcarning set" and then applied as a single standard for all subjects. Although the reported correlations have been excellent by both groups, there is a major theoretical objection in that chest thickness varies tremendously from patient to patient. The "average" attenuation correction approach is therefore subject to serious error in individual patients and is not acceptable for routine clinical care. The approach may still have merit as a research tool to evaluate group data or to compare serial studies in the same patient.

It should also be noted that for all of the count-based volume techniques, a tacit assumption is made that the "true" net ventricular count can be calculated from the image. This implies that all counts arising from the ventricle are included in the region of interest and that background correction is accurate. Unfortunately, there is no direct means of proving either assumption.

Calculation of "relative" ventricular volume is less controversial. This is accomplished quite simply by comparing the net ventricular count rate at selected points in the cardiac cycle before and after an intervention. Since the net count rate is proportional to volume, and the attenuation factor for a given patient remains the same. the change in net count is directly proportional to change in volume. This technique has been used to evaluate volume response to interventions such as leg exercise or the administration of pharmacologic agents. When measurements with ${ }^{99 \mathrm{~m}} \mathrm{Tc}$ 
are made more than five minutes apart, radioactivity decay correction must be applied. The major limitation of the "relative" volume approach is that it does not permit calculation of other derived parameters such as cardiac output and does not allow comparison of absolute ventricular volume in the same subject studied at two different times or between different subjects. Recent data also suggest that hemoconcentration during exercise may cause errors on the order of $5 \%$. Nonetheless, useful estimates of volume change during a sequence of interventions obtained during one setting are provided.

The same considerations noted in the preceding paragraphs apply to ventricular stroke volume. A specific application of relative stroke volume in the left and right ventricles has been described in patients with valvular insufficiency. ${ }^{28-30}$ Since the change in net ventricular counts between end diastole and end systole is directly proportional to volume change an $\mathrm{LV} / \mathrm{RV}$ stroke index ratio (SIR) can be computed directly from the respective stroke counts ("nuclear stroke volume").

$$
\text { SIR }=\frac{\text { LV Stroke Counts }}{\text { RV Stroke Counts }}
$$

Rigo and others ${ }^{28-30}$ have presented very good correlations between the nuclear stroke index ratio calculations and catheterization results. In the normal subject, the stroke index ratio should be 1.0. In actual practice, some variation is encountered due to chamber overlap and the inability to determine net counts in the two ventricules with absolute accuracy.

Although the ejection fraction remains the most important quantitative parameter derived from the ventricular time activity curve further analysis is quite straight forward and several additional parameters are receiving attention.
Fig. 3. (a) Background corrected ventricular time activity curve beginning at end diastole (expressed as $100 \%$ ). Each subsequent point is expressed as a percentage of end diastole. $y$-axis is labeled "volume" to indicate the direct relationship between net ventricular radioactivity and ventricular volume. (b) Graph of first derivative of ventricular time-activity curve providing a measure of peak emptying and filling (indicate to left of $y$-axis). average emptying and filling and the times at which peaks are reached.

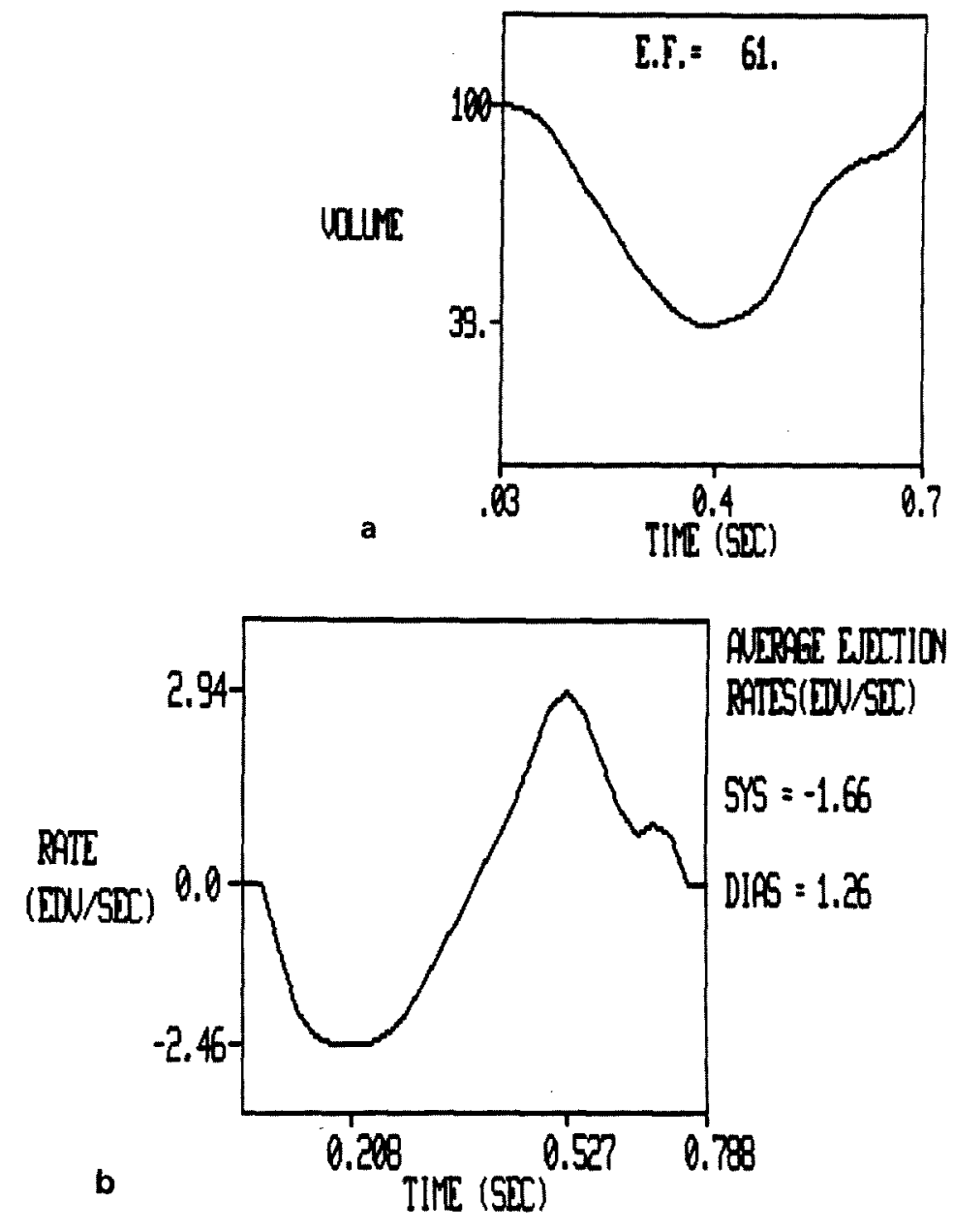


Systolic and diastolic time intervals and rates of filling and emptying are calculated directly from the curve as illustrated in Fig. 3. Maximum and average ejection and filling rates $(\mathrm{dV} / \mathrm{dt})$ are computed by taking the first derivative of the time activity curve at each point. For these measurements to be valid, particularly the peak rates, the time activity curve must generally be filtered. Otherwise, the first derivative amplifies any statistical noise in the data, while smoothing the data loses the high frequency component. There are various ways to overcome these problems. One approach is to fit a polynomial function at each point in the curve prior to obtaining the derivative. Early commercial programs for calculating " $\mathrm{dV} / \mathrm{dt}$ " did not take this step which has caused unreliable results. Therefore, before this type of analysis is used clinically in a given laboratory the method of calculation should be determined and critically appraised. The clinical role of these parameters awaits further definition and study. Early reports suggest that peak emptying and filling rates may become abnormal sooner in the course of coronary artery disease than abnormalities in the ejection fraction and may permit diagnosis from ventriculograms obtained at rest. If this is borne out by further experience it could eliminate the need for stress testing in some patients. Diastolic parameters are also proving useful to assess therapeutic response to various drugs, including calcium blockers, where the ejection fraction may remain the same but changes in filling rate can be measured.

Functional or "parametric" images are twodimensional representations of derived parameters (parametric images). For example, the "stroke volume" image or "difference" image is obtained by subtracting the end systolic image point by point from the end diastolic image. The relative intensity at each pixel location in the ventricle is proportional to the change in count volume occurring at that point (Fig. 4). The normal stroke volume image of the left ventricle has a horseshoe appearance with high intensity at the periphery indicating uniform contraction between end diastole and end systole. Peripheral segments with diminished contraction demonstrate reduced intensity in the image corresponding to the lack of volume change. The stroke volume image has been used to aid in detection of

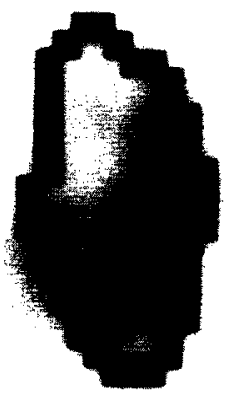

Fig. 4. "Storage volume" image (ED-ES) from a normal subject. The computer detected end diastolic left ventricular border is superimposed for reference.

wall motion abnormalities. The ejection fraction image is a further refinement of the stroke volume obtained by dividing the stroke volume image point by point by a background corrected end diastolic image. ${ }^{23}$ Thus, the intensity at each point is proportional to the ejection fraction at that point.

The "paradox" image as described by Holman and co-workers ${ }^{31}$ is a corollary of the stroke volume image wherein the end diastolic frame is subtracted from the end systolic frame. In normal subjects, this subtraction step results in a void over the region of the left ventricle because the end diastolic counts are higher than the end systolic counts. In patients with ventricular dyskinesis or paradoxical motion, small areas of activity remain in the paradox image where they project outside the end diastolic outline (Fig. 5). Ordinarily paradoxical motion is readily detected using cinematic display techniques and the paradox image is of limited clinical value. Although none of the derived or functional images has found widespread clinical application, they indicate the potential for image manipulation on the digital computer.

\section{FOURIER PHASE ANALYSIS}

Fourier phase analysis is designed to reduce the four-dimensional data from an equilibrium gated blood pool study into an easily interpretable pair of two dimensional images clearly demonstrating subtle regional differences in cardiac contractility (amplitude image) and the sequence of contraction (phase image) of each of thc cardiac chambers. Fourier analysis is essen- 
ANT

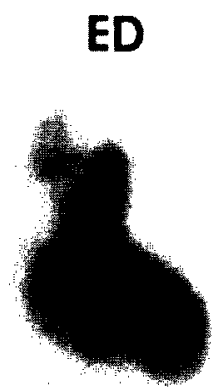

a

LAO

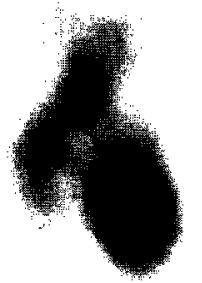

b

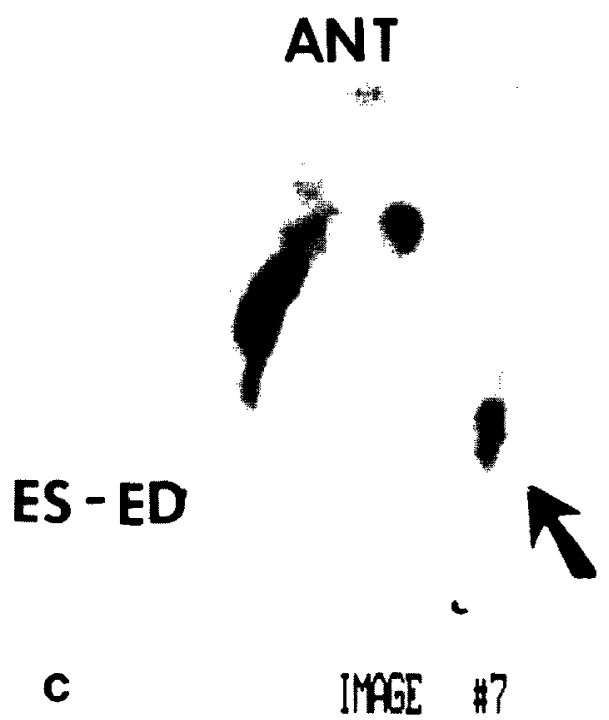

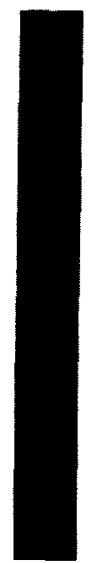
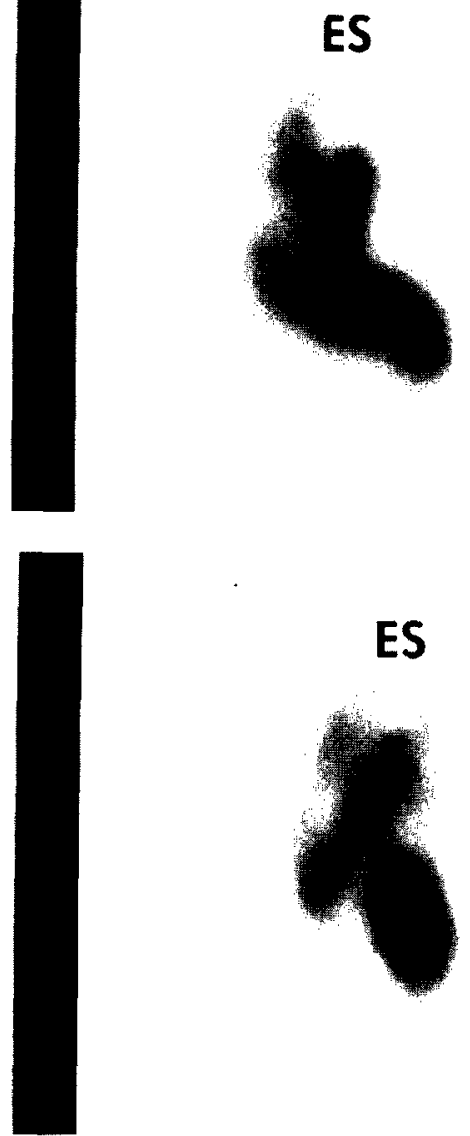

ES
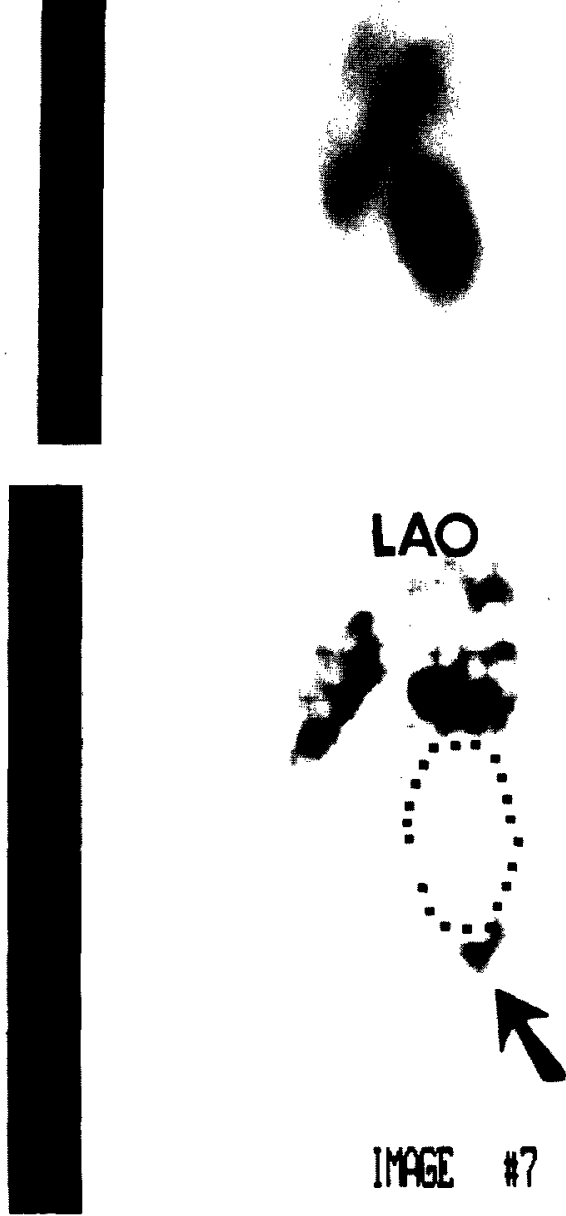

Fig. 5. Anterior (a) and left anterior oblique (b) views at end diastole and end systole in a patient with a prior myocardial infarction and apical dyskinesis. Corresponding "paradox" images in the two views (c) clearly demonstrate the area of dyskinesis (arrows) or foci of residual activity. 
tially a method of mathematically describing any wave form as a series of sine and cosine waves having various amplitudes and phases. In the 1970 s, German investigators ${ }^{32}$ realized that the time activity curve from a cardiac study closely resembled a periodic wave and found that more than $90 \%$ of the information it contained could be condensed into the phase and amplitude characteristics of the first harmonic (cosine wave) derived using the Fourier analysis. By calculating the phase and amplitude of this cosine wave on a pixel by pixel basis, a phase and amplitude map of the cardiac activity can be constructed. The derived phase map is often displayed as a color image in which the color then represents the temporal sequence of cardiovascular chamber emptying. It is displayed beside the phase histogram which plots the number of pixels against the phase angle. Another display mode takes the form of a propagating wave front that sweeps through the image linking pixels with similar phase angles together. It usually starts with the earliest and finishes with the latest phase angles and can be set up in a repetitive mode.

To identify the cardiac chambers, both phase and amplitude images (or modified stroke volume image) are inspected. As the background pixels have small amplitudes and random phase angles, they can be readily excluded from the display by excluding all of the pixels with a low amplitude (less that 10\% of maximum amplitude).

The acquisition of equilibrium data for this analysis requires some modification of the standard techniques. Parameters that need to be considered include: (1) optimal chamber separation using a modified left anterior oblique projection with $20^{\circ}-$ $30^{\circ}$ caudal tilt; (2) at least 28 frames per cardiac cycle; (3) image magnification (1.5-2 times standard imaging value); and (4) temporal and spatial smoothing of the image. Ideally, the study should be acquired in list mode so that very tight R-R intervals can be chosen. In practice, however, because the contraction sequence examines the changes of pixels relative to neighboring pixels, the last 2-4 frames of the study can be eliminated from analysis thereby overcoming the problem of data drop-out.

The application of phase analysis includes cardiac chamber separation and cdge detcction.
Because the atria contract approximately $180^{\circ}$ out-of-phase when compared to the ventricles, they are usually separated from these latter structures. The lateral borders of the ventricles can be separated from the background activity because the background activity lacks periodicity. Limitations of this method include the difficulty of separating right from left ventricular chambers and the atria from the great vessels because they have very similar periodicities.

One major use is looking at conduction abnormalities in a situation where a close electromechanical link has been demonstrated. ${ }^{33-34}$ The normal cardiac contraction sequence is shown in Fig. 6. Note the earliest areas of contraction occur in the region of the septum of the right ventricle and left base. Then there is uniform and simultaneous activation of both ventricles. This sequence closely agrees with the known electrophysiologic sequences of ventricular activation. This strong electromechanical link allows Fourier phase imaging to map bundle branch blocks, ${ }^{35}$ pacemaker tip sites and sometimes foci of ventricular tachycardia where the heart is known not to contain any major scars. In this laboratory, ${ }^{34}$ it has been used to lateralize accessory bypass tracts from the knowledge that these bypass tracts cause early contraction of neighboring myocardial fibers and therefore result in abnormal areas of early activation. Using esophageal pacing, ${ }^{36}$ it has been found that as electrical pre-excitation is increased (as seen by larger delta waves on the ECG) the area of early abnormal emptying segments is also increased and vice versa (Fig. 7). Furthermore, patients with concealed bypass tracts have been shown to have appropriately abnormal phase images despite a normal ECG at time of data acquisition. Further work in this area is continuing in an attempt to assess the meaning of the various contraction patterns seen in association with the surface electrocardiographic findings.

The third application is examining global and regional contraction abnormalities as an index for identifying latent coronary artery disease. ${ }^{32-3 i}$ Studies have demonstrated subtle tardo kinesis in myocardial regions supplied by diseased coronary arteries, even in the presence of apparent normal systolic emplying. This abnormality is usually demonstrated by an abnormal (delayed) phase in comparison to neighboring ventricular 
Fig. 6. Emptying sequence of normal heart: the heart requires an electrical signal to begin contraction. Three areas of the heart activated almost simultaneously. These areas are represented by the blue color.

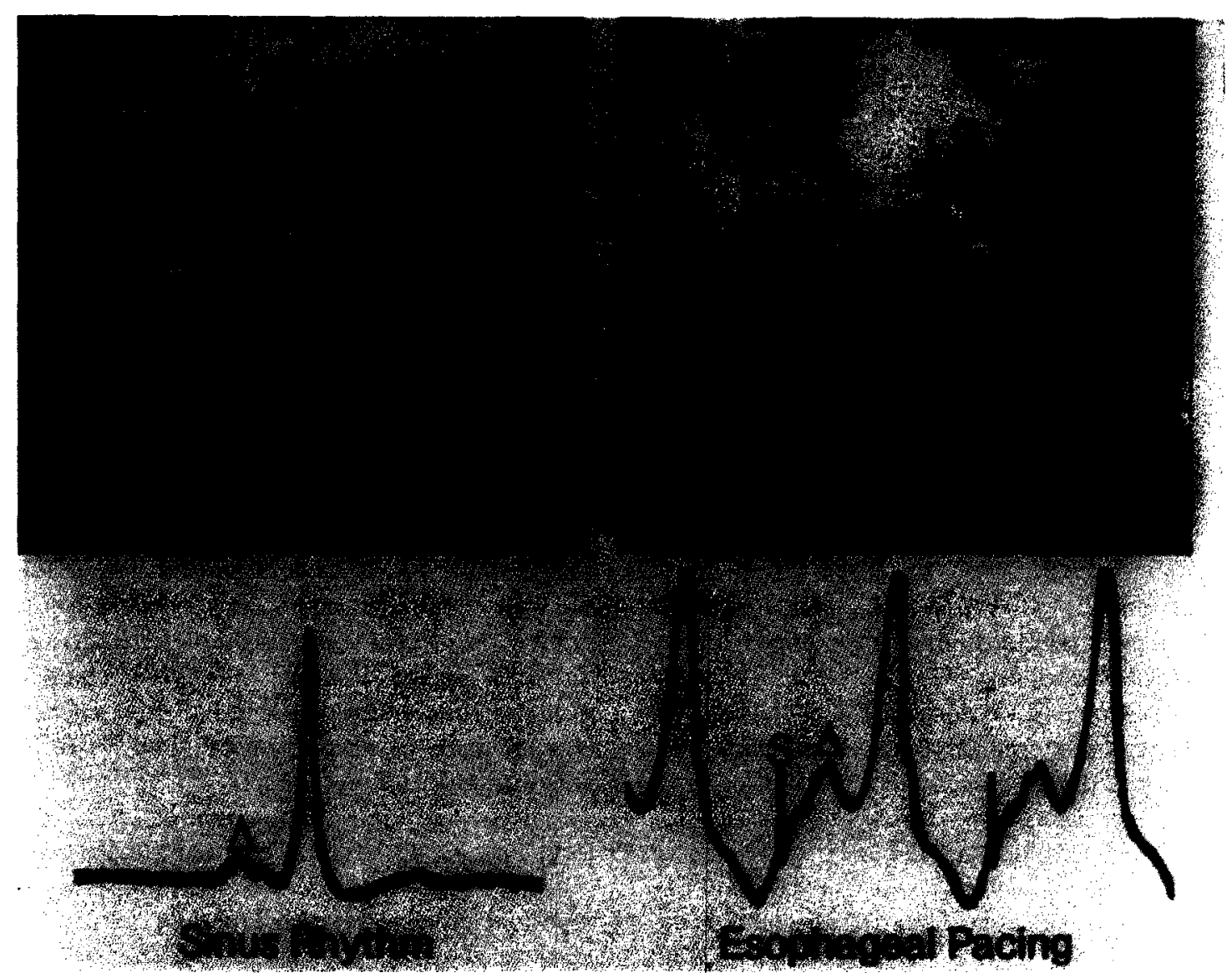

Fig. 7. A case of Wolff-Parkinson-White syndrome with a left-sided accessory bypass track. An early emptying segment (EI) is seen during sinus rhythm where a small delta wave $(\Delta)$ is present. Early emptying is also seen in the intraventricular septum (IVS) where normal conduction occurs. During esophageal pacing, the ectopic early emptying septum (EI) has increased in size in the early phase of the septum (IVS) are absent. The accompanying ECG shows that conduction is almost completely through the accessory pathway as evident from the large delta wave $(\Delta)$ in the bizarre QRS complex. (S $=\mathbf{s t i m u l u s}$ artifact from esophageal pacemaker.) 
segments even if the amplitude image may be normal. Less subtle abnormalities of myocardial contraction are usually detected by an abnormality in both the amplitude of contraction (amplitude image) and corresponding phase shift. Although this is a very sensitive technique, it may not be very good at localizing the region of abnormality because it represents a volume abnormality rather than a tangential motion abnormality (the best method of localizing regional dysfunction).

Contraction abnormalities are known to also occur in patients who have a combination of conduction abnormality and myocardial contractility depression due to areas of ischemia or infarction. The phase and amplitude image might help differentiate this problem particularly in patients who have left bundle branch block. The usual left bundle branch block picture demonstrates a uniform but delayed contraction of the left ventricle in comparison to the right ventricle. ${ }^{35}$ Despite the delay in contraction, the phase angle limits for the entire left ventricle are very narrow. Therefore, regions within this ventricle that are out-of-phase are abnormal and can be inferred to be due to localized depression of myocardial function as seen in coronary artery disease.

Although this imaging technique has much potential for the assessment of various parameters of ventricular function, it is still essentially qualitative in nature. Future work in this area is needed for quantifying the size and assessing the clinical significance of the variation and magnitude of these abnormalities.

\section{SERIAL MODE ACQUISITION}

The serial (or list) mode data acquisition is a viable alternative to the frame (or image) mode acquisition previously described. In list mode acquisitions the $(x, y)$ coordinate of each detected gamma ray is stored in computer memory in a temporal sequence along with a $1-10$-msec time marker and a physiologic time marker defining the onset of cardiac systole. The latter marker is derived from an $\mathrm{R}$-wave trigger device that recognizes the occurrence of each QRS complex on a standard ECG that is being recorded during radionuclide imaging.

Serial mode data acquisition is utilized in two ways. The first is as an ECG gated first pass study, ${ }^{37.39}$ A compact bolus of radionuclide tracer is rapidly injected into a large peripheral vein and the ECG as well as radionuclide data are collected for the $25-35 \mathrm{sec}$ that it takes for the tracer to traverse each of the four cardiac chambers. Since each gamma-ray event requires a full computer word to specify its location, the usual amount of data generated from a single crystal gamma camera with a count rate of $40,000-60,000$ counts/sec will take up to two megabytes of digital storage. Once collected, the data can be reformatted into a series of images to create a dynamic display of the tracer transit. From this, the time intervals for which the tracer is in the right or left ventricle, may be identified. The data are then reformatted to create a single composite cardiac cycle from the 3-5 cardiac cycles during which the tracer is in the right ventricle and the 8-12 cycles when the isotope is in the left ventrielc. The various global and regional cardiac function parameters such as ejection fraction, mean filling and emptying rates, and ventricular volumes can now be calculated in a similar manner to that employed for the equilibrium gated method. ${ }^{37-39}$

The advantages and disadvantages of ECG gated first pass studies are defined in Table 2. Briefly, the major advantage is that each cardiac chamber can be viewed in the right anterior oblique view (the single most important projection in cardiac imaging) with little interference from background activity or chamber overlap because the bolus occupies only one chamber at a time. This is particularly important for analysis of right ventricular function. The major disadvantages are the relatively low count density and the inability to perform more than two studies because of the radiation dosimetry of the isotope used $\left({ }^{\mathrm{Tc}} 99 \mathrm{~m}\right)$. These disadvantages are the main impetus for the development of short half-life isotopes obtained from a long half-life generator system such as mercury-gold $\left(\mathrm{Hg}^{195 \mathrm{~m}}-\mathrm{Au}^{195 \mathrm{~m}}\right)$ and osmium-iridium ( $\left.\mathrm{Os}^{191}-\mathrm{Ir}^{191 \mathrm{~m}}\right)$.

The second major application of serial mode acquisition is with the radioisotope already equilibrated in the red blood cell pool. For quantitative analysis of ventricular function, radionuclide data need to be acquired in the modified left anterior oblique view. Data storage, however, is exactly the same as during the ECG gated first pass study. ${ }^{7}$ Therefore, for the two minutes nec- 
Table 2. Comparison of Cardiac Radionuclide Acquisition Techniques

\begin{tabular}{|c|c|}
\hline Advantages & Disadvantages \\
\hline \multicolumn{2}{|c|}{ Equilibrium Gated Studies } \\
\hline Multiple studies from 1 dose of isotope & Needs at least $2 \mathrm{~min}$ of time for data acquisition \\
\hline High count density in ventricle & Quantitative analysis only from one view (LAO) \\
\hline Require only a modest amount of data & Data degradation by arrhythmias \\
\hline Computer mernory & $\begin{array}{l}\text { Careful background correction is necessary because of low } \\
\text { left ventricular to background ratio }\end{array}$ \\
\hline No preprocessing (reformatting) of data prior to analysis & RV function difficult to analyze because of RA overlap \\
\hline \multicolumn{2}{|l|}{ Can estimate valvular regurgitation } \\
\hline \multicolumn{2}{|c|}{ Serial Mode During ECG Gated First Pass Studies } \\
\hline Needs only $20-30 \mathrm{sec}$ for data collection & $\begin{array}{l}\text { Only two studies can be performed because of radionuclide } \\
\text { dosage limitation }\end{array}$ \\
\hline Ventricles can be viewed in RAO projection & Low count density in ventricular region of interest \\
\hline Optimal method for RV analysis & Require extensive reformatting before analysis \\
\hline \multicolumn{2}{|l|}{ High target to background ratio low background activity } \\
\hline \multicolumn{2}{|l|}{ Can also be used for shunt detection } \\
\hline \multicolumn{2}{|c|}{ Serial Mode Collection with Blood Pool Labeling } \\
\hline Handles most arrhythmias & $\begin{array}{l}\text { Only } 25 \text { min of data acquisition because of computer mem- } \\
\text { ory limits }\end{array}$ \\
\hline High count rate density giving good statistical certainty & $\begin{array}{l}\text { Careful background correction as for equilibrium gated stud- } \\
\text { ies }\end{array}$ \\
\hline $\begin{array}{l}\text { Can set varying } R-R \text { limits depending on needs for diastolic } \\
\text { function }\end{array}$ & Reformatting twice as long as acquisition \\
\hline
\end{tabular}

essary to acquire sufficient radionuclide data to adequately assess global and regional ventricular function, an inordinate amount of computer memory or data storage space is required since the typical count rate is $15,000-30,000$ counts/ sec: Over 7.0 megabytes of memory are often necessary. This major handicap precludes this acquisition method from being used in the routine situation in most institutes. It is, however, the technique of choice in evaluating the ventricular function of patients with frequent ectopic beats. ${ }^{34,40}$

Prior to acquisition, the frequency of the ectopic beats is noted and radionuclide data are collected for the time necessary to give $2 \mathrm{~min}$ of cycles which are sinus beats. For example, in trigeminy, $1 / 3$ of the cycles are sinus, the other $2 / 3$ consisting of the premature and postextrasystolically potentiated beats. Thus, 6 min of radionuclide data need to be acquired. To minimize the storage space necessary, lead shielding is used to exclude as much extracardiac activity as possible.

Once data are acquired, the computer searches through the raw data and constructs a histogram, grouping all cardiac beats with similar R-R intervals. For the patient in trigeminy, the histogram shows three groups of cardiac cycles (Fig. 8). By inspecting the ECG, the group with the shortest $R-R$ interval represents the interrupted sinus beats. By setting the R-R interval limits about this group of beats, the computer reformats a single composite cardiac cycle which excludes all but the interrupted sinus beats. Previous studies have demonstrated that the
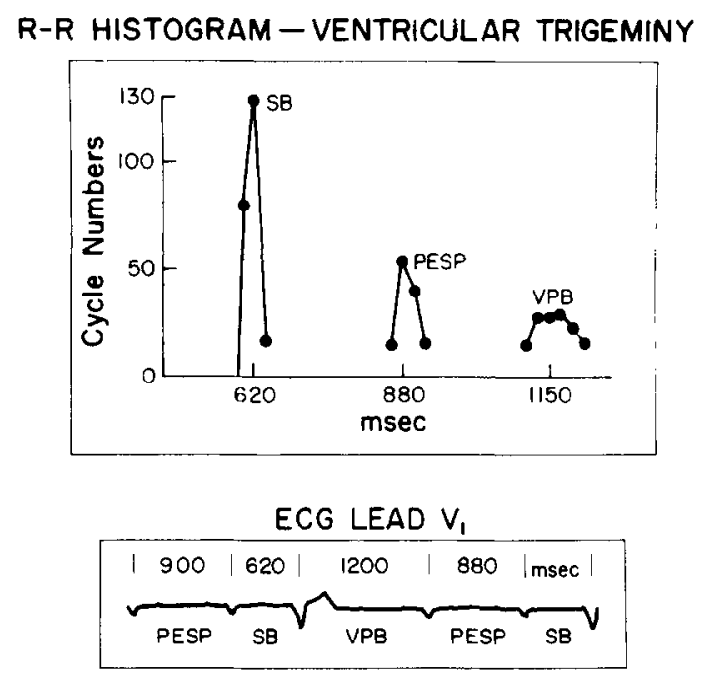

Fig. 8. The R-R interval histogrem for a patient in ventricular trigeminy shows three distinct groups of cardiac cycles; a sinus beat (SB), post-extrasystolically potentiated (PESP) beat, and ventricular premature beats (VPB). 
sinus beat function during frequent ectopic beats varies little from the function of the ventricle when the rhythm is sinus. Therefore, standard analysis methods of the reformatted sinus beat will give representative assessment of baseline left or right ventricular function.

In cases other than trigeminy, the histograms alter in morphology with the center cluster no longer representing just the postextrasystolically potentiated (PESP) beat but a combination of sinus and PESP beats. The computer can separate the sinus beats by setting the $R-R$ limits around this central cluster and then always eliminating the first beat that fulfills the R-R criteria. Examination of the ECG shows that this will nearly always eliminate the PESP beat.

By combining the information in the histogram and the ECG rhythm strip, an algorithm can usually be constructed to separate the sinus beats, and for that matter, the PESP and ectopic beats in most situations. For example, we have performed a study comparing the PESP response to atrial and ventricular premature beats using this technique.

If computer data storage is not a problem, then all intervention such as exercise stress can be acquired in the ECG gated serial list mode, giving the advantages of arrhythmic exclusion, and also the ability to choose very tight R-R intervals at each level of stress. This advantage is important if accurate systolic and diastolic function is required at intermediate levels of stress. Nevertheless, the reformating of such studies, takes a significant enough amount of time (1-2 hr), excluding its use for anything but research purposes.

\section{QUANTITATIVE MYOCARDIAL PERFUSION IMAGING}

More than 20 years ago, Saperstein and coworkers, ${ }^{41}$ recognized that following the intravenous administration of radioactive potassium, the distribution of the tracer in the patient represents the regional blood flow. In the 1960s investigators sought to visualize directly the myocardium with other potassium analogs. Carr et al. ${ }^{42}$ employed Cesium-131 as well as Cesium-129 for myocardial perfusion imaging. Poor physical properties of both these isotopes prohibited their use.
Isotopes of rubidium were tried subsequently,,$^{434}$ and their biologic behavior more closely approximated that of potassium. However, the physical half-lives of rubidium and unfavorable physical properties limited their use as an agent to image myocardial perfusion. In the 1970s, Hurley et al., ${ }^{45}$ described the use of Potassium-43 for imaging the heart. However, it is not an ideal agent in that its gamma photon energies are greater than $300 \mathrm{keV}$ and its short physical half-life of $22 \mathrm{hr}$ limited its commercial distribution.

Kawana et al. ${ }^{\text {th }}$ described the use of thallium in the 1970s and demonstrated that thallium behaved, in many respects, like potassium in biologic systems. Subsequently, Lebowitz ${ }^{47}$ developed a technique of producing Thallium-201 of high purity from thallium nuclides with less favorable imaging spectra.

Thallium-201 is the most widely used myocardial imaging agent because of its favorable biologic and physical properties. The myocardial extraction efficiency is higher than potassium (approximately $85 \%$ ) and the liver uptake is lower. Imaging is acceptable with the $68-80 \mathrm{keV}$ mercury characteristic $x$-rays. A physical halflife of 73 hr permits commercial distribution.

New myocardial imaging agents to be introduced within the next year or so will probably be based on the technetium isotope with the active molecule being of easier preparation and lower cost than thallium.

\section{Image Processing}

The early myocardial perfusion images were interpreted by visual inspection of analog scintiscans taken directly off the gamma camera display. In a high percentage of patients analyzed in this fashion the results were satisfactory and the image findings correlated well with coronary angiography.

In a significant number of cases $(10 \%-20 \%)$, however, the thallium interpretation did not correlate with other modalities. In order to improve the accuracy of interpretation various computer techniques have been proposed. The first technique that was introduced was background subtraction. The average target to background ratio of thallium myocardial images is approximately 2.7 to 1 immediately after exercise. ${ }^{48}$ The sim- 
plest technique for background subtraction was to measure the activity in a region adjacent to the heart and to subtract a constant number from all the pixels within the image. The limitation of the procedure was that it did not account for differential background throughout the region of interest. In 1976, Goris et al..$^{49}$ described an interpolative background subtraction technique which was superior to techniques previously used. The technique starts by defining a rectangular region surrounding the heart with the sides of the rectangle being adjacent to the myocardial surfaces. The background within the circumscribed region is then calculated from the count values measured on the boundary. (See Goris ${ }^{49}$ for description of technique.) The interpolative background technique can correct for the varying background levels throughout an image. Further modifications have been made to this technique.

Although the background subtraction techniques improved the target to background ratios, interpretation still depended upon the subjective impressions of the observer with significant intra- and interobserver variations. In order to address this problem, quantitative computer techniques have been extended to include image analysis.

\section{Quantitative Myocardial Analysis}

Meade et al. ${ }^{50}$ was one of the first to describe computer assisted quantitative analysis using circumferential profiles. This enabled the viewer graphically to display left ventricular thallium activity as a function of regional location and thus permit objective regional comparisons of immediate postexercise and redistribution images.

The process of creating circumferential profiles involves displaying upon the computer screen a thallium image. The image should be smoothed, and background subtracted depending upon the observer's preference. The center and epicardial surface of the left ventricle are defined either using visual or computer critcria. Radii

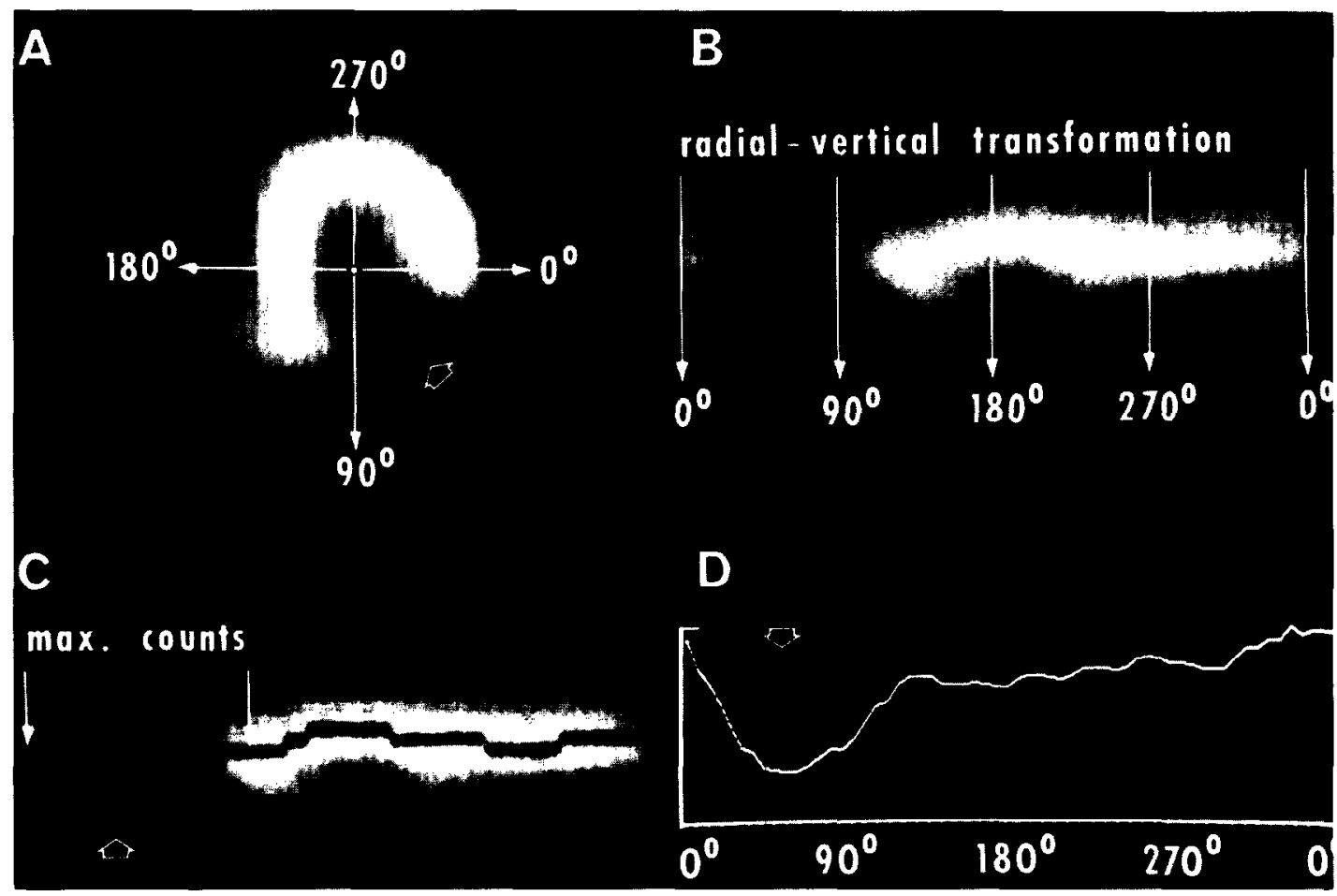

Fig. 9. From the polar coordinate representation (A), a polar/orthagonal or radial/vertical transformation (B) can be displayed. (A) demonstrates an LAO thallium-201 image with an inferoapical perfusion defect. (L denotes deficit.) (B) between the 0 and $90^{\circ}$ region demonstrates the perfusion abnormality. (C) is the same data as in (B) with the addition of maximal myocerdial wall count profile. (D) is the curve of maximal myocardial wall count from (C). (Reprinted with permission of New England Nuclear. ${ }^{\text {92 }}$ ) 


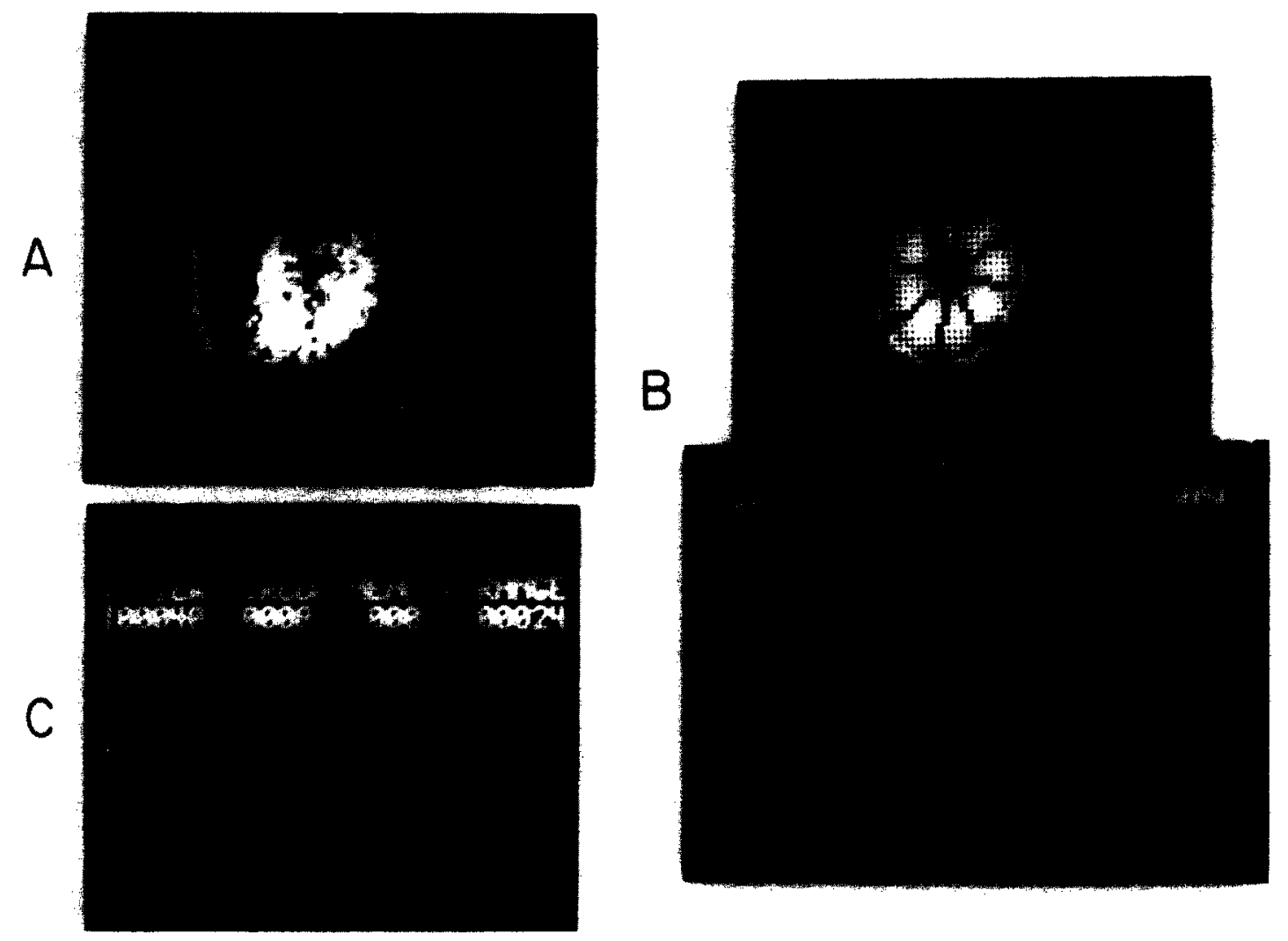

Fig. 10. Circumferential profile technique. In (A) an ellipse is placed around left ventricle (LV) of subject imaged in $60^{\circ} \mathrm{LAO}$ view. In (B) the computer has generated an outside count LV edge and radii from the center of the LV to each circumferential point. In (C), the curve is superimposed on predetermined normal values. The apex is marked for orientation. (Reprinted with permission of Journal of Nuclear Medicine. ${ }^{51}$ )

are constructed from the center of the left ventricle to multiple points on the epicardial surface proceeding in a clockwise fashion using standard polar coordinates.

The regional myocardial distribution of thallium is then determined by calculating the average activity per pixel along each radius, and then normalizing these data to the radius with the highest average activity. A normalized regional uptake of Thallium-201 can then be plotted against an angular position with the epicardial surface being transformed vertically downward. The image can be displayed either as a radial/ vertical or a polar/orthogonal transformation (Fig. 9). The maximal pixel values along each radius can also be defined and a circumferential profile or maximal pixel count isocontour of the left ventricular myocardium can also be displayed (Fig. 10). The principal advantage of the circumferential profile technique is that it permits simple quantitative evaluation of thallium images which are relatively free from intraobserver and image quality variability.

The following is a discussion of further retinements of circumferential profiles. Meade ${ }^{50}$ in his paper on quantitative methods in the evaluation of Thallium-201 myocardial perfusion images. described the technique for generating circumferential profiles, but Burow actually coined the term "circumferential profiles" and described his method in $1979 .{ }^{51}$ Burow's method utilized a 9-point smoothing algorithm without background subtraction. The observer selected the central point of the left ventricle and the lengths of the major and minor semi-axes of an ellipse that surrounded and contained the area of the left ventricle. This ellipse was situated to separate the left ventricle from the remainder of the heart and background regions. Based on a threshold percent of maximum supplied by the observer, an iso-count contour was determined by searching from the edge of the ellipse towards 
the center of the left ventricle. A threshold value of $50 \%$ was generally selected first, followed by adjustments up and down until the iso-contour approximated the outer edge of the left ventricle as assessed visually. The centroid part of the iso-count contour was then determined and radii constructed to each point on the iso-count contour (normally 70-100 points). The distribution of thallium activity was determined by calculating the average activity per pixel along each radius and the data normalized to the radius with the highest average activity. These data were then displayed as a "circumferential profile" curve plotting normalized thallium activity against angular position. Circumferential profiles for all the views of both the immediate and delayed images were generated. This technique allowed a quantitative method for evaluating the images, but did not take into account the changes in activity as a function of time, from initial to redistribution.

A report by Garcia et al. ${ }^{52}$ in 1981 described space/time quantification of Thallium-201 myocardial scintigraphy. Garcia's work is perhaps one of the most sophisticated techniques for quantitative computer analysis. The technique began by compensating for tissue crosstalk by performing bilinear interpolative background subtraction as described by Goris et al. Garcia modified Goris' technique by the use of a proximal weighting factor as described by Watson et al. ${ }^{53}$ This modification was performed to produce a more rapid falloff of the computed tissue crosstalk, this being important where the boundary of the rectangle surrounding the myocardium contained areas of higher uptake such as the liver. After background subtraction the images were smoothed using a standard 9-point algorithm. Circumferential maximal-count profiles of the myocardial distribution of thallium were then obtained (Fig. 11). The profile is constructed by the computer from the values of 60 radii spaced at $6^{\circ}$ intervals plotted clockwise. These profiles quantify the segmental activity as an angular function referenced from the visually located center of the left ventricular cavity. The operator also assigns the maximum radius to which the computer will search. This is done to prevent the algorithm from searching outside the left ventricular myocardium into other structures such as the right ventricle. These circumferential profiles are then plotted relative to the scintigraphic apex. The computer automatically shifts the points so that the apical location on the circumferential profile coincides with $90^{\circ}$. The curves are normalized to the maximal pixal value found in any of the profiles.

Garcia also computed washout in the circum-

\section{ALGORITHM}
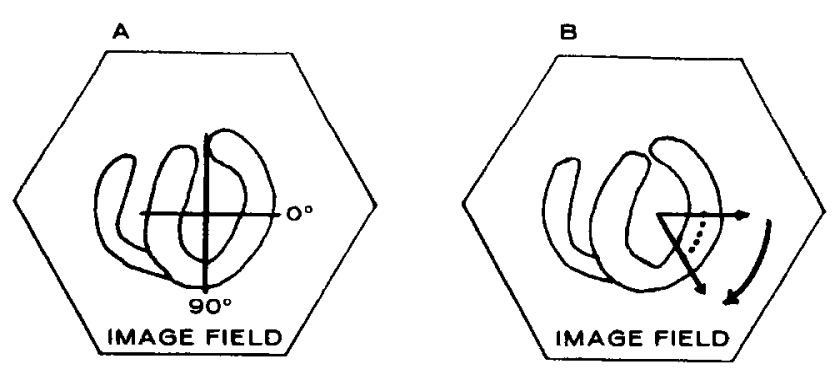

Fig. 11. Diegrammatic representation and method of obtaining circumferential profiles of the myocardium. All the coordinate reference axes are shown in (A). Pixels for circumferential profile analysis are found by performing a radial search for maximum value at $6^{\circ}$ intervals (B) throughout $360^{\circ}$. The maximum value shown as black dots (B) and (C) are then replotted in (D) for each angle as a percent of the maximum value for the circumferential profile. Top curve and left (D) represents circumferential profile for stress thallium-201 image; below, that from the 4-hr delay image. (Reprinted with permission of Journat of Nuclear Medicine. ${ }^{62}$ )

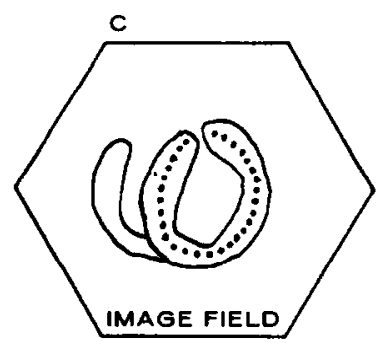

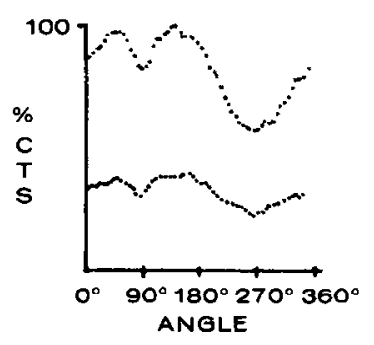


ferential profiles as a percent change from stress to $40 \mathrm{~min}, 4$ and $24 \mathrm{hr}$. Normal washout parameters were established in a group of patients without coronary artery disease. The normal values were calculated by measuring a mean effective half-time as calculated in each of the 60 angular locations within the myocardium. Two standard deviations as calculated by a one-tailed analysis encompassed $97.5 \%$ of the population. The $t_{1 / 2}$ values were then used when analyzing patients by identifying patients whose regional $t_{1 / 2}$ fell outside of two standard deviations from the normal population.

Based on these parameters, Garcia et al. ${ }^{52}$ determined the following criteria for abnormality: (1) an initial defect as defined by an $18^{\circ}$ segment (three contiguous radii) of the stress profile falling below the normal limit; and (2) slow washout as defined by an $18^{\circ}$ segment (three contiguous radii) of the 4-hr washout profile falling below the normal limits. To be considered abnormal, the patient needed at least two abnormal $18^{\circ}$ arcs in the initial distribution and washout profiles pooled from all three views.

Subsequent to Garcia's report, Watson et al. ${ }^{54}$ described a spatial and temporal quantification of planar thallium myocardial images. This technique differed from Garcia's work in that rather than obtaining a circumferential profile. profile slices were obtained through the heart in the standard views (Fig. 12). A computer manipula- tion of the images was required to identify position and alignment so that initial and delayed images could be compared. This technique divided the heart into five segments, which could be compared both spatially and temporally. Normal washout kinetics were determined from normal patients and the normal slopes per segment were identified. Patients could be classified as abnormal if their slopes fell above or below two standard deviations of the normal values.

The final word on the ideal technique for quantitative thallium analysis is not in at this time. Newer techniques continue to be developed and evaluated to reduce intraobserver variability and increase the sensitivity and specificity of thallium myocardial scintigraphy. The techniques as described by Garcia and Watson show great promise but both lack critical testing in prospective clinical study.

\section{EMISSION TOMOGRAPHY}

The recently developed radionuclide techniques described earlier have contributed to the noninvasive diagnosis and management of cardiovascular disease. Although these procedures have allowed the convenient and accurate measurement of cardiac function as well as relative determination of myocardial perfusion under a variety of physiologic conditions, they suffer from several shortcomings.

With standard planar imaging, all activity in the organ of interest as well as underlying and

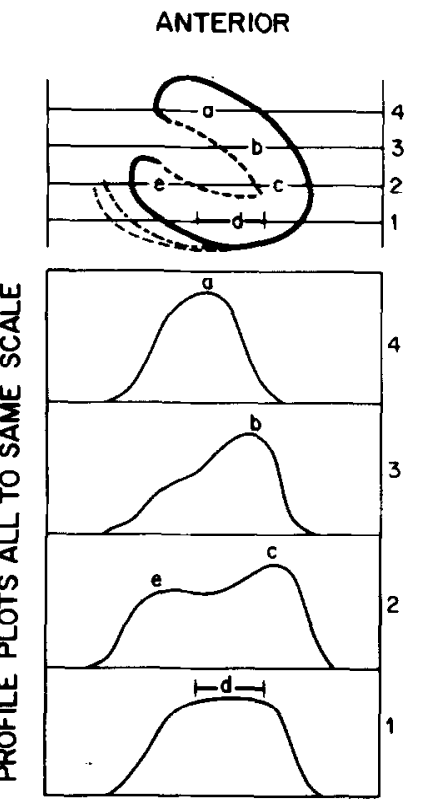

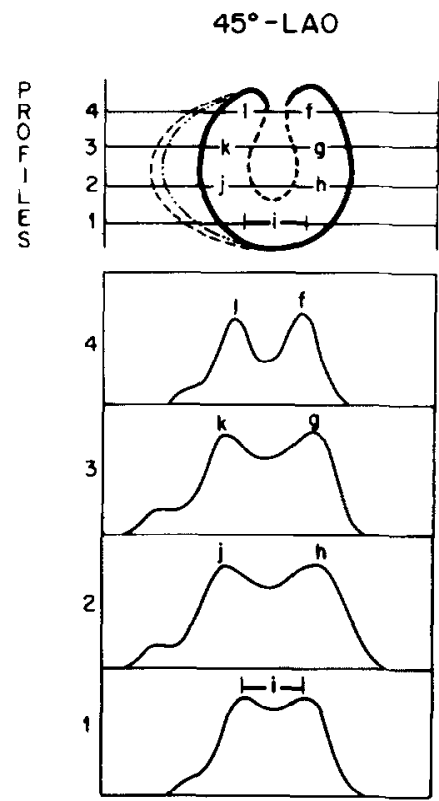

Fig. 12. Illustrating placement of profile slices on net myocardial images. Profiles below are appropriately numbered and lettered for reference. Peaks characterize thallium-201 behavior. (Reprinted with permission of Journal of Nuclear Modicine. ${ }^{53}$, 
overlying background activity from outside the organ is projected into a single image. This not only reduces image contrast which can obscure important details of organ anatomy, but renders accurate quantitative measurements practically impossible since all knowledge of the distribution of background activity is lost. These problems can be greatly reduced using tomographic imaging techniques which are capable of measuring the distribution of radionuclides in the body in a full three-dimensional fashion. With tomographic imaging, serial slices through the long axis of the heart may be obtained which provide a means of viewing the three-dimensional distribution of the tracer as a set of two-dimensional images. This not only allows the separation of the tracer from foreground and background structures, but also provides a more accurate localization of the tracer within the myocardial segments. Tomographic imaging may further provide a means of accurately measuring ventricular chamber volumes, the size of infarcted or ischemic segments, perfused myocardial mass, and the quantity of tracer per unit of myocardial tissue. With the refinement of tomographic imaging as well as development of new radiopharmaceuticals, it may be possible to noninvasively measure regional myocardial blood flow as well as metabolic rates.

Over the past five years, a number of tomographic methods have been investigated for obtaining three-dimensional information about the distribution of radioisotopes, particularly $\mathrm{Tl}$ 201 , in the heart. In order to understand the pros and cons of various tomographic methods, some understanding of the basis for constructing a three-dimensional image from two-dimensional projections is necessary.

Well before our present sophisticated understanding of three-dimensional imaging evolved, focal plane tomography was employed in radiology to obtain depth-specific information. In this method, the $x$-ray tube and film were moved in a coordinated manner so that the $x$-ray shadows from only one plane in the patient were stationary on the film and the other planes were blurred out. Much effort was devoted to determine what pattern and extent of motion produced the smoothest blurring so that "out-of-focus" objects produced minimal interference with the "infocus image plane."

In nuclear medicine, one of the earliest methods of obtaining threc-dimensional information ${ }^{55}$ was described by Kuhl who, in essence, backprojected views taken from a number of angles onto an image plane. In both of these methods, information from out-of-focus regions was only "diluted" by blurring it out over a larger area and it was not until methods for accurately removing the blurred information were introduced that quantitative, three-dimensional imaging in radiology or nuclear medicine was achieved.

In 1917, while searching for solutions to gravitational equations, the Austrian mathematician, Radon, showed that it is mathematically possible to reconstruct a density function from its projections. ${ }^{56}$ It was not until 1956 that this result was rediscovered, refined, and applied to radioastronomy by Bracewell. ${ }^{57}$ Applications of threedimensional image reconstruction techniques to electron microscopy by DeRosier and Klug (1968), ${ }^{58} \mathrm{x}$-ray tomography by Cormack (1963, 1964), ${ }^{59,60}$ and Hounsfield (1973), ${ }^{61}$ and to nuclear medicine by Chesler (1971), ${ }^{62} \mathrm{Kuhl}^{63}$ and Kay and Keyes ${ }^{64}$ followed. The essence of this work is that if the proper set of measurements is made, it is possible to reconstruct the three-dimensional image without superimposed defocused information. Some excellent summaries of the reconstruction process may be found in references 65,66 , and 67 .

In nuclear medicine, a conventional planar image is a sum of all the activity along rays perpendicular to the camera face. Such a sum is a projection of the radiotracer distribution onto the plane of the camera face when self attenuation is ignored. For this reason, the Radon integral alluded to above can be used to relate these projection images to the volume distribution of isotope.

Without delving into the underlying theory, it can be simply stated that in order to reconstruct properly a three-dimensional object distribution, one must have measured projections of this object onto a plane with views taken over a minimum angular range of $180^{\circ}$. The minimum number of views required in $180^{\circ}$ is determined by the size of the object and the resolution of the imaging system. This number is approximately equal to the circumference of the object divided by the resolution. Thus, a $30-\mathrm{cm}$ diameter chest imaged with $2-\mathrm{cm}$ resolution requires

$$
\frac{\pi \cdot(30)}{2}=50 \text { views } / 180^{\circ} \text {. }
$$




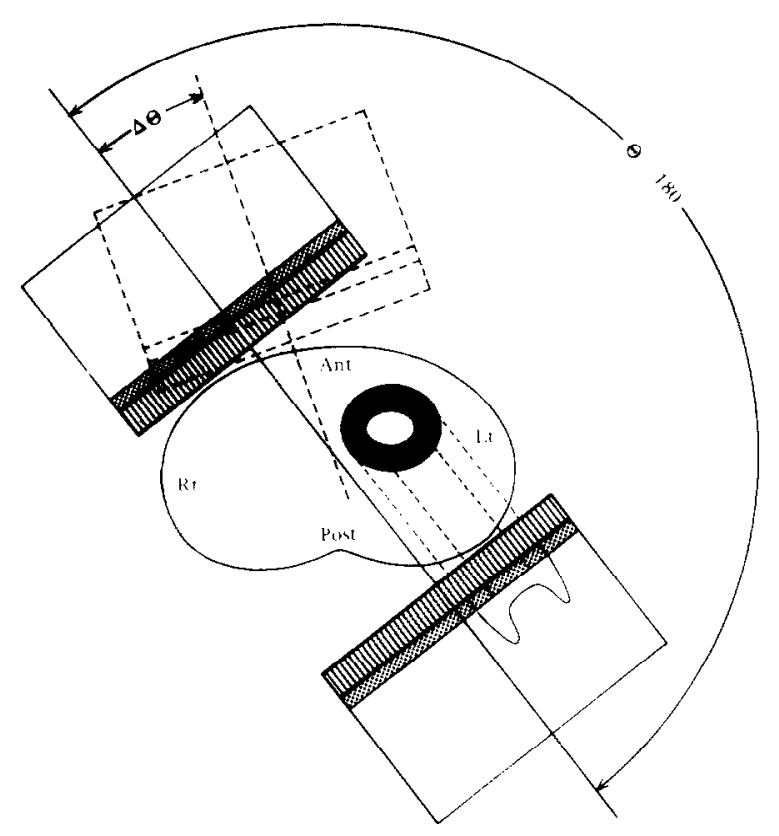

Fig. 13. Illustration of rotating gamma camera tomography of the heart. Camera acquires images over the $180^{\circ}$ arc from right anterior oblique to left posterior oblique in steps of $\Delta \theta$. A profile of the intensity distribution on the camera face is indicated schematically.
It should be emphasized that practical implementation of the three-dimensional imaging process virtually requires a digital computer. The data are acquired as digital images, the deblurring or reconstruction operation is accomplished digitally, and the final images are displayed with a computer and may be interpreted quantitatively with a computer.

A gamma camera mounted so it can be rotated about a patient may be used to acquire a set of images over at least a $180^{\circ}$ range with any desired angular interval between images. This satisfies the sampling requirements described earlier so that accurate, distortion free, threedimensional images can be reconstructed from such a data set. For this reason, rotating camera tomography of the heart will be described first and may be used as a basis of comparison for other techniques employing slant hole collimators and seven pinhole apertures. Positron imaging systems and single photon ring detectors are not yet widely available for clinical application and will therefore not be discussed.

\section{Rotating Gamma Camera Tomography}

The first commercially available camera designed for single photon emission tomography was the General Electric 400-T, a wide fieldof-view camera, counterbalanced and mounted on a large ring bearing so it can be motor driven about a patient. The geometry is shown schematically in Fig. 13. Virtually all of the major camera manufacturers are now offering a rotating camera for acquisition of tomographic data. Reconstruction and display software is available from at least five commercial sources.

At our institution, tomographic T1-201 imaging is performed with a GE 400T using a high efficiency, low energy collimator. Imaging begins 20 min following a $2-\mathrm{mCi}$ dose of thallium and the total acquisition time is 20 mint.

Although sampling the object over $180^{\circ}$ ideally provides enough information to unambiguously reconstruct the object, when self-attenuation and loss of resolution with distance from the collimator are considered. better images are obtained for some organs by acquiring data over $360^{\circ}$ and averaging the opposed views. In the case of the heart, especially when using thallium-201 with its relatively low energy gamma ray, it has been found that for a given imaging time, better images are obtained when data are acquired over a $180^{\circ}$ arc from RAO to $I . P O$ than when the full $360^{\circ}$ arc is sampled. This is because the heart is primarily located in the left anterior quadrant of the body, and data obtained when the camera is rotating through the right posterior quadrant suffers severely from attenuation by midline structure and poor resolution because of the increased distance of the heart from the 
collimator. This is illustrated in Fig. 14, which shows images reconstructed from an anterior $180^{\circ}$ arc and a posterior $180^{\circ}$ arc with the same total imaging time in each case.

Figure 15 illustrates three of the possibilities for image display. The first set of images is generated directly by the reconstruction algorithm and is oriented transverse to the long axis of the patient. The slices are viewed from the feet and displayed in caudal to cephalad order. Images in the second set of images are vertical slices taken parallel to the long axis of the heart while those in the third set are transverse to the long axis of the heart. These last two image sets are generated by stacking up the first set of images and reslicing them along the desired axis using the computer. ${ }^{68}$ The transverse section slices are more difficult to interpret because they transect the heart at an odd angle to its symmetry axis. This angle also varies from patient to patient. The short axis slices illustrate perfusion abnormalities in a far more intuitive manner except at the apex where it is sometimes difficult to tell whether the apex is infarcted or whether the slice is partially outside of the myocardium. long axis slices give continuous contours through the apex and show apical defects well.

A more detailed discussion of rotating camera tomography and current application to the heart may be found in references. ${ }^{69-71}$

\section{Restricted View-Angle Systems}

Several methods which do not sample the object over a full $180^{\circ}$ have been described for tomographically imaging the heart. These include a rotating single segment slant hole collimator, ${ }^{72.73}$ a double rotating slant hole collima- tor, ${ }^{74}$ a quadrant slant hole collimator, ${ }^{75-77}$ and seven pinhole collimators. ${ }^{78-80}$ All of these systems require computers for data acquisition and for image reconstruction. These systems which fall under the general heading of restricted viewangle systems will be briefly described below. Table 3 summarizes their performance characteristics.

\section{Rotating Slant Hole Collimators}

An Anger camera equipped with a parallel channel collimator whose channels are not perpendicular to the camera face may be used to view an object from different directions merely by rotating the collimator on the face of the stationary camera as illustratcd in Fig. 16. Views are also acquired at intermediate rotation angles. Muehllehner reported use of this approach for tomographic imaging in $1970 .{ }^{81}$ The images were essentially reconstructed by backprojecting the multiple views onto a series of image planes located at different distances from the camera face and no attempt was made to remove the blurred data. Application of slant hole collimators to cardiac imaging with the addition of digital image reconstruction methods has more recently been described by Gottschalk. ${ }^{72}$

It can be seen from Fig. 16 that the slant angle and camera diameter jointly determine the commonly viewed volume. Slant hole collimators are generally constructed with a $25^{\circ}$ slant for standard $25-\mathrm{cm}$ diameter cameras and $30^{\circ}$ for large field-of-view cameras. The maximum range of viewing angle is seen to be just twice the slant angle so it is evident that a full $180^{\circ}$ arc is not sampled. The effect of the missing views on reconstructed image quality has been reported by
Fig. 14. T1-201 myocardial images obtained with a rotating gamma camera showing the difference in image quality when data is acquired over a $180^{\circ}$ anterior arc (a) and a $180^{\circ}$ posterior arc (b) with a fixed imaging time.

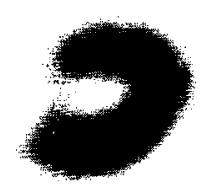

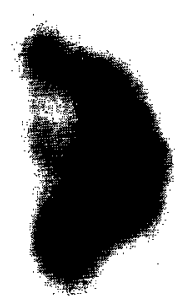

b 


\section{a}
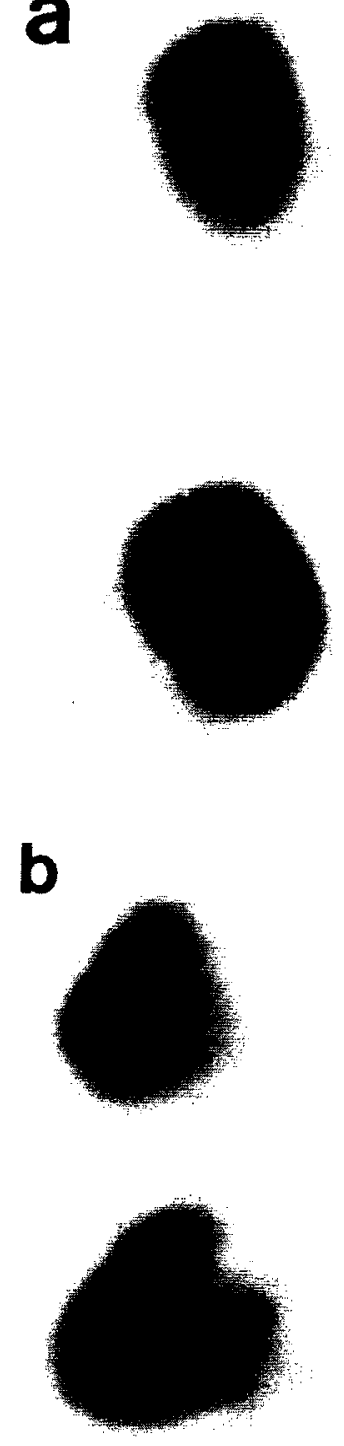
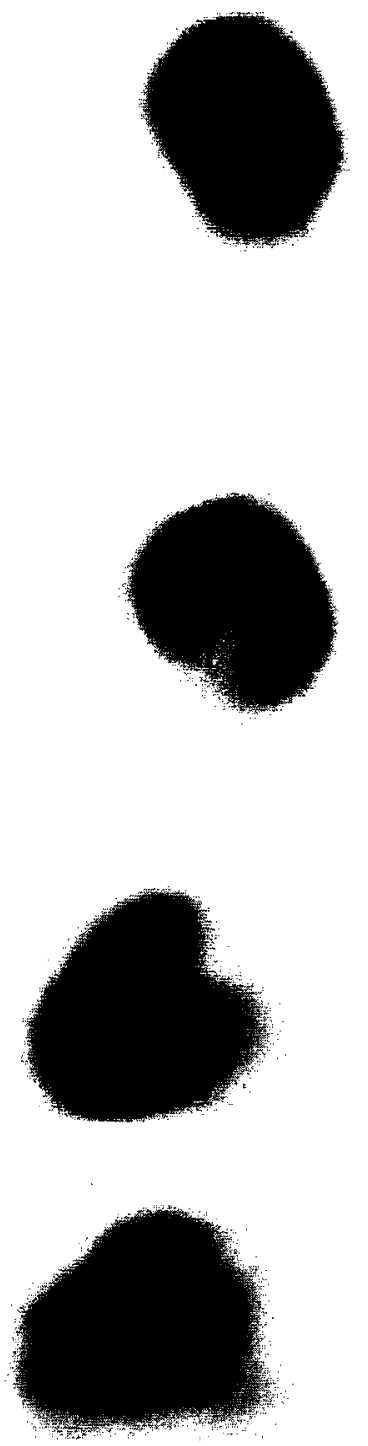

Fig. 15. Comparison of various tomographic slice orientations for T1-201 myocar dial imaging. (a) Transverse to long axis of patient viewed from patient's feet. (b) Vertical slices parallel to long axis of heart viewed from left posterior. (c) Transverse to long axis of heart viewed from apex.

several authors. ${ }^{82-85}$ Figure 17 is from the work of Koral ${ }^{85}$ who reconstructed images of an $8-\mathrm{cm}$ diameter spherical shell of activity from simulated data for a $26^{\circ}$ slant angle collimator used with a $25-\mathrm{cm}$ diameter camera. The collimator was rotated over $360^{\circ}$ in $30^{\circ}$ steps. The top row illustrates three slices through the sphere taken at the indicated distance from the central axis. The bottum row shows reconstructed images in planes perpendicular to the camera face. Usually rotating slant hole images are reconstructed in planes parallel to the camera face in which they appear as circular donuts. The reorganization of

these images into perpendicular slices simulates the long axis slices illustrated in Fig. 15 and is done here to illustrate the distortion arising from limited angle sampling. Note that the sphere is elongated and the intensity at what would be the apical region of the heart is greatly diminished relative to the equatorial plane. Higure 18 , from the work of Budinger, ${ }^{84}$ not only demonstrates the apical wash-out as above but shows that when an elongated nonspherical object simulating the myocardium is imaged with the camera face not perpendicular to the long axis, that the apical defect moves laterally on the long axis 

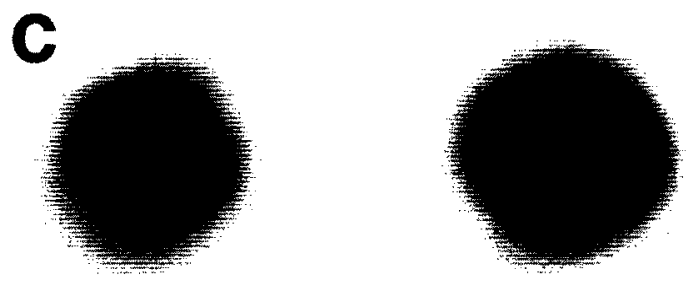

Fig. 15. Continued
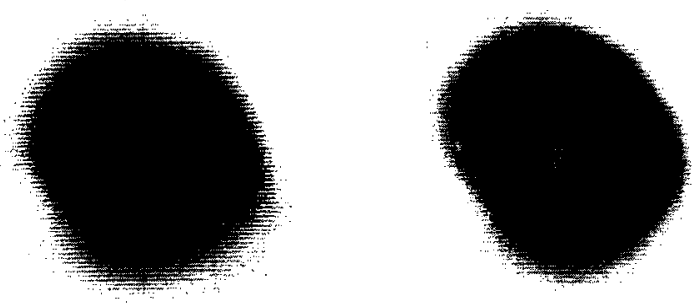

images and will, therefore, show up in short axis slices as a perfusion deficit. Furthermore, this figure illustrates that the severity of these artifacts is related to the amount of missing information. A $90^{\circ}$ sampling interval yields appreciably better results than a $60^{\circ}$ sampling interval. References 72,73 , and 76 describe experimental phantom results with a slant hole collimator which confirm these simulations. The apparent perfusion deficit resulting from camera misalignment is a major difficulty in all of the restricted angle tomographic techniques and diligent effort is required on the part of the technologist who sets up the study to position the camera perpendicular to the long axis of the heart to within $\pm 15^{\circ}$. This positioning is particularly difficult with a single segment slant hole collimator because several images must be acquired and compared for different collimator rotation angles to ascertain that the long axis is perpendicular to the camera.

Another problem arising from the limited viewing angle is the cross talk between reconstructed planes. The depth resolution function is wider and has longer tails than the in-plane resolution function so that a defect which exists in one plane of the object will appear in several planes above and below the actual location. Furthermore, activity in front of and behind the defect shows up in the plane of the defect and

reduces the contrast of the defect. For example, a $100 \%$ cold defect in the Iowa Heart Phantom ${ }^{86}$ is reconstructed as only a $45 \%$ defect in the work reported by Gottschalk. ${ }^{72}$ In a conventional planar image of the same phantom, the defect shows up as a $20 \%$ defect. Thus, limited angle tomography in this instance increases the defect contrast more than a factor of two but falls short of giving a quantitatively accurate result.

\section{Multisegment Slant Hole Collimators}

The field-of-view has a different shape for multisegment slant hole collimators as shown in Fig. 19, but the more efficient use of detector area gives approximately a factor of 2 and 4 increase in count rate for the 2 and 4 segment collimators compared to the single segment collimator. Some of this increased efficiency may be traded for a collimator design with improved resolution. When used with a small crystal camera, however, Ref. 75 indicates this field-of-view is too small to image enlarged hearts. The additional views, particularly for the quadrant slant hole collimator, greatly facilitate orienting the camera perpendicular to the long axis of the heart since the camera angle can be adjusted until all four images appear symmetric. Nevertheless, the fundamental problems of limited angle tomography described above apply equally well to the multisegmental slant hole collimators. 
68

FROELICH ET AI

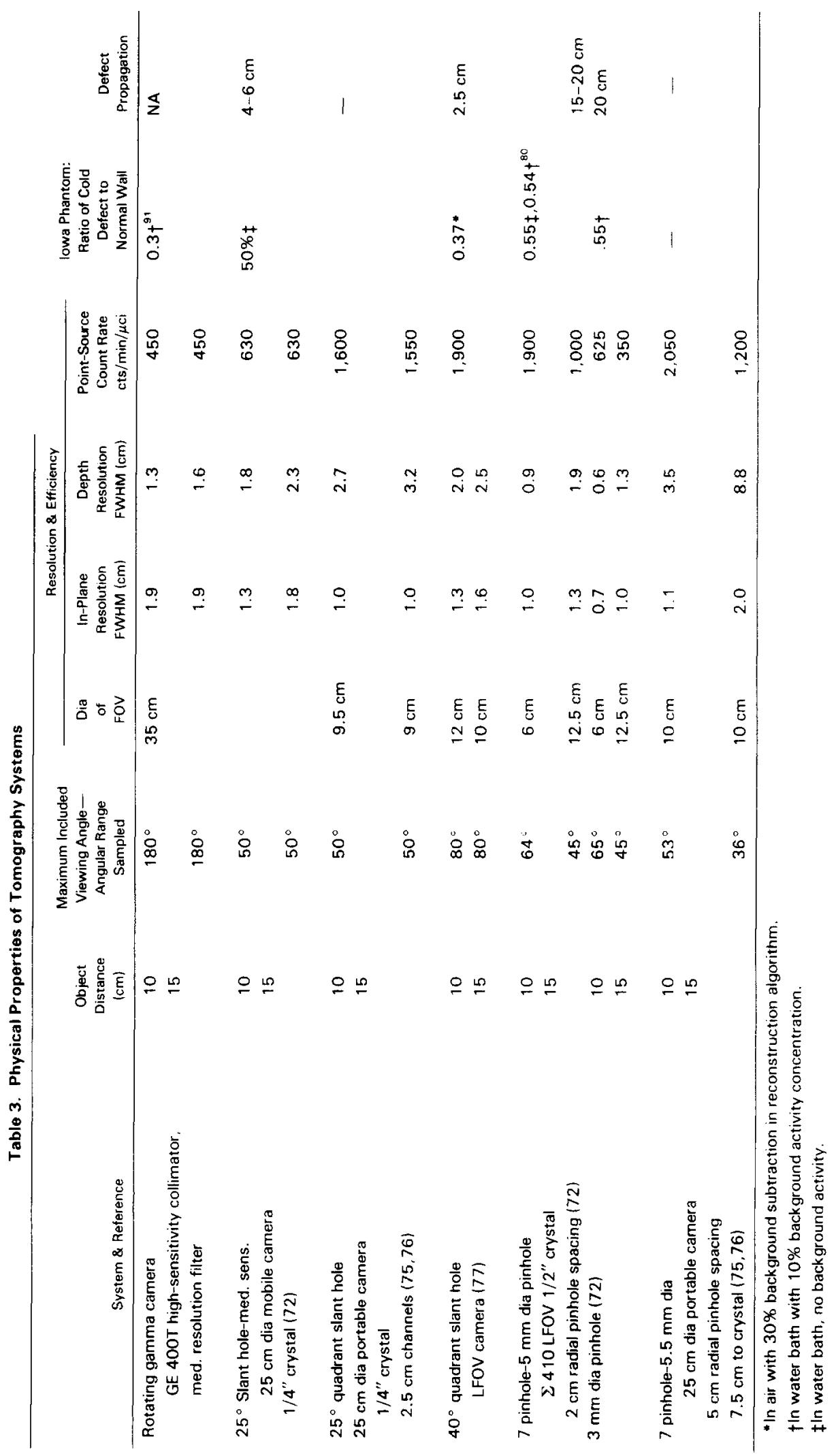




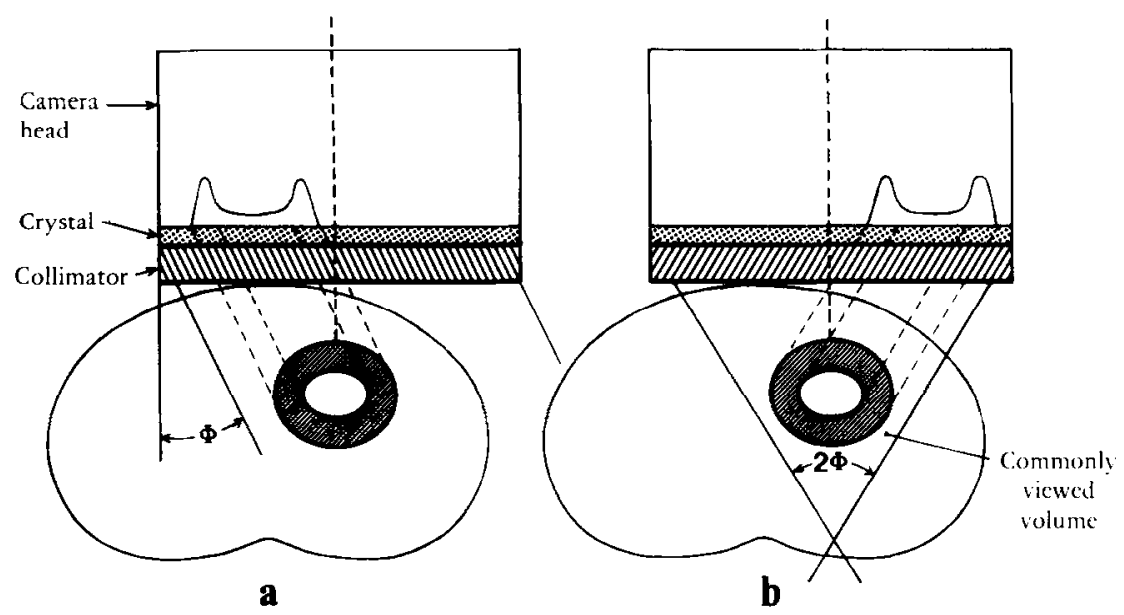

Fig. 16. Geometry for single segment rotating slant hole collimator. The collimator slant angle is $\phi$. In (b) the collimator is shown after it has been rotated $180^{\circ}$ on the camera face. The angle between the two views is $2 \phi$. Usually 8 to 12 views per $360^{\circ}$ of collimator rotation are acquired.

In order to improve the limited depth response, Chang ${ }^{77}$ constructed a $40^{\circ}$ slant hole collimator for a large field camera. As shown in Table 3, this collimator gives a depth response of about 2 -cm full width at half maximum (FWHM) but with a field-of-view found to be too small for about $10 \%$ of the patients.

\section{Seven Pinhole Aperture}

An array of pinholes can also be used to acquire images from different angles for tomographic reconstruction as illustrated in Fig. 20.

Fig. 17. Illustration of effects of restricted viewing angle on reconstructed images of spherical shell. Computer simulated data for a rotating slanthole collimator is used. Slices are reconstructed in planes perpendicular to camera face to illustrate distortion inherent in limited angle tomography. Had slices been reconstructed in planes parallel to camera face, the images would appear as doughnuts and the image elongation and decreased apical intensity would not have been clearly portrayed.

Object images
The pinhole aperture differs markedly from any of the slant hole collimators.

1. Reconstruction is performed from only seven images which are all acquired simultaneously. This permits dynamic data acquisition.

2. As opposed to parallel hole, slanted collimators, the angular range over which points in the object are viewed, varies with distance from the aperture. This causes the depth response to vary markedly over the object depth.

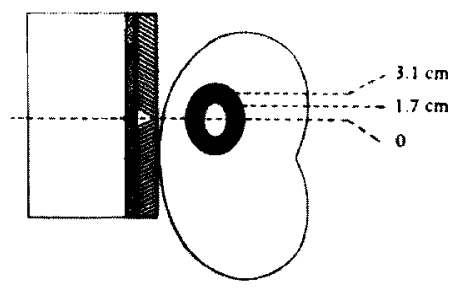
0
$1.7 \mathrm{~cm}$
$3.1 \mathrm{~cm}$
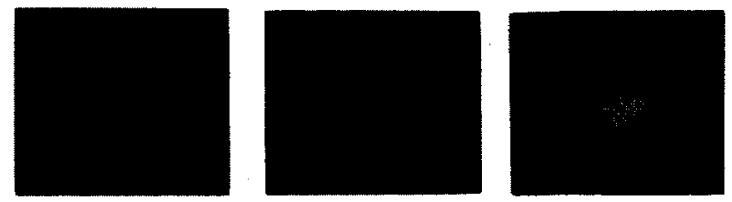

Reconstructed
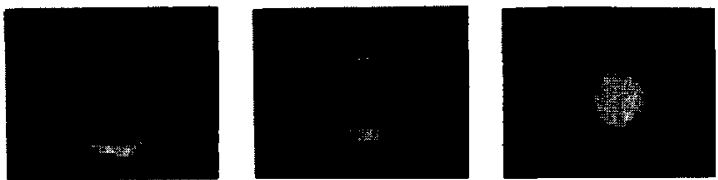

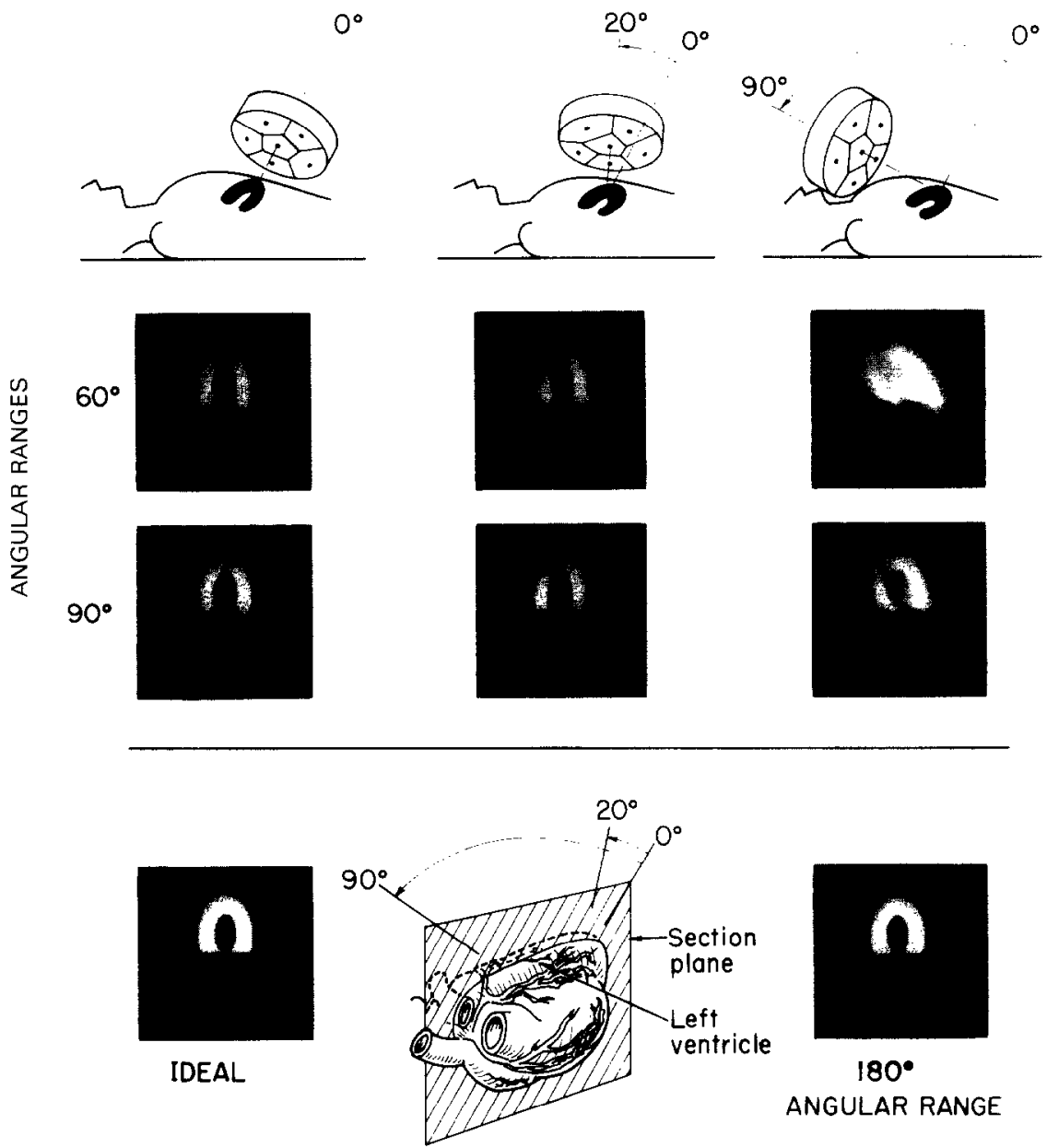

Fig. 18. Illustration of dependence of reconstructed image quality on orientation of a tomographic imaging system relative to symmetry axis of object and on the angular range sampled. Optical axis is varied from $0^{\circ}$ to $90^{\circ}$ relative to symmetry axis and angular range of views is varied from $60^{\circ}$ to $180^{\circ}$. (Courtesy of T.F. Budinger and Journal of Nuclear Medicine. ${ }^{84}$ )

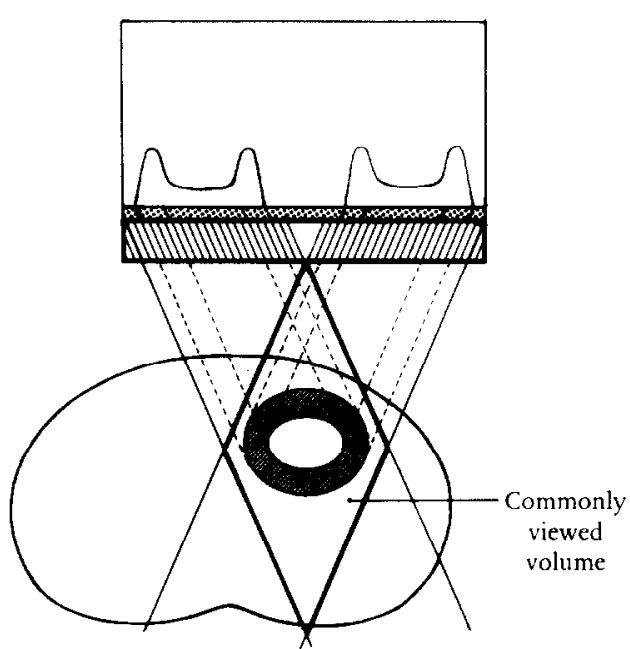

Fig. 19. Field-of-view for a multisegment slant hole collimator.
3. Point source efficiency varies nearly as $1 / \mathrm{r}^{2}$ with source distance from the aperture.

4. The sampling density is lower than for slant hole collimators in which 8 or 12 views are taken. $\Lambda$ point source defocuses into seven point images and somewhat greater structure is contributed by out-of-focus activity.

5. Because the object can be minified by increasing object distance, larger objects can be imaged with the pinhole aperture albeit with reduced efficiency and resolution.

From Table 3, it can be seen that the biggest difference between seven pinhole and slant hole tomography is distance over which the defect in the cardiac phantom propagates. It varies from about $2 \mathrm{~cm}$ for a $40^{\circ}$ slant hole ${ }^{77}$ to more than 20 $\mathrm{cm}$ for the seven pinhole aperture. ${ }^{72,75,80}$ This is 
Fig. 20. Multi-pinhole geometry showing (a) the field-of-view and (b) change in angular range that is sampled for object points at different distances from the collimator.

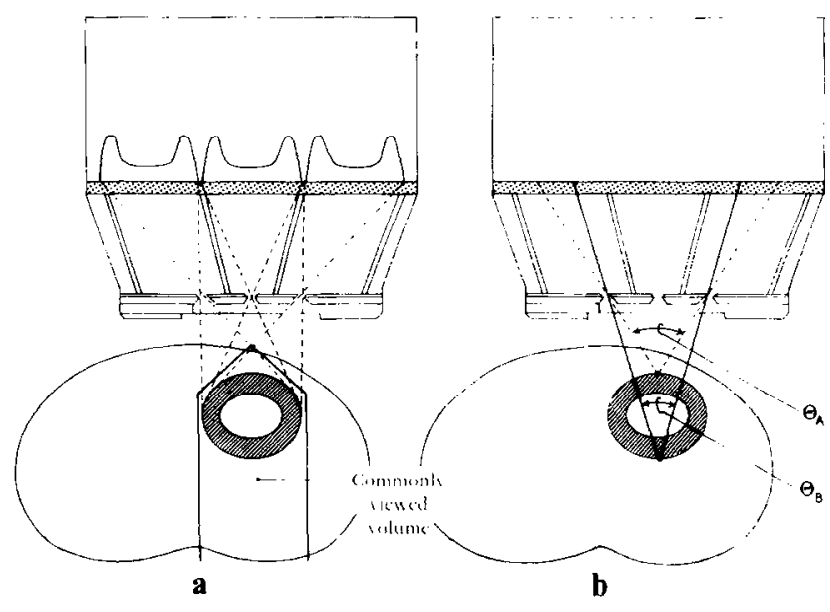

principally caused by the reduction in viewing angle with distance for the pinhole aperture. Large differences in performance are evident in depth resolution between a seven pinhole system designed for a large field camera and a small field portable camera. The small field portable system gives depth resolution at midheart of about $4-5 \mathrm{~cm}$ while the large field system gives depth resolution of $1-2 \mathrm{~cm}$.

\section{SUMMARY}

Gamma-camera tomography gives uniform in-plane and cross-plane resolution with propagation of the defect from one plane to another determined only by the usual camera-collimator resolution. These images may be reformatted by computer to portray slices in any orientation. Cross plane resolution is slightly worse than for the other methods, but it is uniform. The efficiency is less than either quadrant slant hole or seven pinhole apertures. Rotating cameras are not portable nor can they be used for dynamic studies.

Both slant hole collimators and seven pinhole apertures distort the object in the depth dimension because of the limited viewing angle. The slant hole geometry provides somewhat better sampling and less plane-to-plane cross talk especially for the more distant planes. The full width half maximum of the depth response is not a sensitive indicator of this problem because the depth response function has very long tails.

To date, best overall performance of the limited angle methods is offered by the $40^{\circ}$ slant hole collimator on a large field-of-view camera. This, however, is not a portable unit and has a field-of-view too small for about $10 \%$ of the patients.

Seven pinhole imaging offers the advantage of having been well studied by a number of institutions. ${ }^{87}$ A A large pool of normal patient studies exists and the performance is well documented. Although the seven pinhole alone is suited to dynamic studies, any of the methods may be adapted to multigated studies.

\section{REFERENCES}

1. Zaret BL, Strauss HW, Hurley PH, et al: A noninvasive scintiphotographic method for detecting regional ventricular dysfunction in man. $\mathbf{N}$ Engl $\mathbf{J}$ Med 284:1165, 1971

2. Strauss HW, Zaret BZ, Hurley PJ, et al: A scintiphotographic method for measuring left ventricular ejection fraction in man without cardiac catherization. Am J Cardiol 28:575, 1971

3. Mullins CB, Mason DT, Ashburn WL, et al: Determination of ventricular volume by radio-isotope-angiography. Ain J Cardiol 24:72, 1969

4. Secker-Walker RH, Resnick L, Kunz H, et al: Measurement of left ventricular ejection fraction. J Nucl Med 14:798, 1973

5. Parker JA, Secker-Walker R, Hill R, et al: A new technique for the calculation of left ventricular ejection fraction. J Nucl Med 13:649, 1972

6. Burow RD, Strauss HW, Singleton R, et al: Analysis of left ventricular function from multiple gated acquisition cardiac blood pool imaging. Comparison to contrast angiography. Circulation 56:1024, 1977

7. Green MV, Ostrow HG, Douglas MA, et al: High temporal resolution ECG-gated scintigraphic angiocardiography. J Nucl Med 16:95, 1975

8. Strauss HW, Singleton R, Burow R, et al: Multiple gated acquisition: An improved noninvasive technique for evaluation of regional wall motion and left ventricular function. Am J Cardiol 39:384, 1977

9. Holman BL, Parker JA: Computer Assisted Cardiac 
Nuclear Medicine. Boston, Little, Brown and Company, 1981

10. Liberman DE (ed): Computer Methods. St. Louis, C.V. Mosby Co., 1977

11. Rollo DF (ed): Nuclear Medicine Physics, and Agents. St. Louis, C.V. Mosby Co., 1977

12. Royal HD, Brown PH, Claunch BC: Mobile gamma cameras: A comparative evaluation. Radiology 128:229-234. 1978

13. Folland FD, Hamilton GW, Larson SM, et al: The radionuclide ejection fraction: A comparison of three radionuclide techniques with contrast angiography. J Nucl Med 18:1159, 1977

14. Green MV, Brody WR, Douglas MA, et al: Ejection fraction by count rate from gated images. $J$ Nucl Med 19:880, 1978

15. Schelbert HR, Verba JW, Johnson AD, et al: Nontraumatic determination of left ventricular ejection fraction by radionuclide angiography. Circulation 51:902, 1975

16. Brady TJ, Lo K, Thrall JH, et al: Exercise radionuclide ejection fraction: Correlation with exercise contrast ventriculography. Radiology 132:703-705, 1979

17. Berger HJ, Matthay RA, Loke J, et al: Assessment of cardiac performance with quantitative angiocardiography: Right ventricle ejection fraction with reference to findings in chronic obstructive lung disease. Am J Cardiol 41:897, 1978

18. Maddahi J, Berman DS, Matsuoka DT, et al: A new technique for assessing right ventricular ejection fraction using rapid multiple-gated equilibrium cardiac blood pool scintigraphy: description, validation and findings in chronic coronary artery disease. Circulation 60:581-9, 1979

19. Slutsky R, Hooper W, Gerber K, et al: Assessment of right ventricular function at rest and during exercise in patients with coronary heart disease; a new approach using equilibrium radionuclide angiography. Am J Cardiol 45:639, 1980

20. Holman BL, Wynne J, Zielonka JS, et al: A simplified technique for measuring right ventricular ejection fraction using the equilibrium radionuclide angiocardiogram and the slanthole collimator. Radiology 138:429-35, 1981

21. Bodenheimer MM, Banka VS, Fooshee CM, et al: Detection of coronary heart disease using radionuclide determined regional ejection fraction at rest and during handgrip exercise: correlation with coronary arteriography. Circulation 58:640-8, 1978

22. Maddox DW, Wynne $J$, Uren $R$, el al: Regional ejection fraction; A quantitative radionuclide index of regional left ventricular performance. Circulation 59:10019,1979

23. Maddox DE, Wynne J, Uren R, et al: Regional ejection fraction; a quantitative radionuclide index of regional left ventricular performance. Circulation 41:12308,1978

24. Slutsky R, Karliner J, Ricci D, et al: Ventricular volumes by gated equilibrium radionuclide angiography; a new method. Circulation 60:556-64, 1979

25. Dehmer GJ, Lewis SE, Hillis LD, et al: Nongeometric determination of left ventricular volumes from equilibrium blood pool scans. Am J Cardiol 45:293-300, 1980

26. Links JM, Becker LC, Shindledecker JG, et al: Measurement of absolute left ventricular volume from gated blood pool studies. Circulation (in press).
27. Gibbons RH, Lee JL. Cobb FR, et al: Ejection fraction response to exercise in patients with chest pain and normal coronary arteriograms. Circulation 64:952-957, 1981

28. Rigo P, Alderson PO, Robertson RM, et al: Measurement of aortic and mitral regurgitation by gated cardiac blood pool scans. Circulation 60:306-312, 1979

29. Sorenson SG. O'Rourke RA. Chaudhuri TK: Noninvasive quantitation of valvular regurgitation by gated equilibrium radionuclide angiography. Circulation 62:1089-1098 1980

30. Lam W, Pavel D, Byrom E, et at: Radionuclide regurgitant index: value and limitations. Am $J$ Cardiol 47:292-298, 1981

31. Holman BL, Wynn J. Idoine J, et al: The paradox image: A non-invasive index of regional left ventricular dyskinesis. J Nucl Med 20:1237-1242, 1979

32. Adam W, Terkowska A, Bitter T, et al: Equilibrium gated radionuclide ventriculography. Cardiovasc Radiol 2:161-173, 1979

33. Links JM. Douglas KM, Wagner MN: Patterns of ventricular emptying by Fourier analysis of gated blood pool studies. J Nucl Med 21:978-892, 1980

34. Chan W, Kalff V, Rabinovitch MA, et al: Noninvasive mapping of accessory $\mathrm{A} / \mathrm{V}$ bypass tracts. Proceedings, First International Symposium of Nuclear Cardiology, Tel Aviv. Israel, 1981 (in press)

35. Botvinick E, Dunn R, Frais $M$, et al: The phene image Its relationship to patterns of conduction and contraction Circulation 65(3):551-561, 1982

36. Chan W, Kalff V, Dick M: The use of Fourier phenc mapping techniques in patients with accessory bypass tracts $J$ Nucl Med (Abstr) (in press)

37. Schod N, Nickel O: Assessment of ventricular function with first pass angiocardiography. Cardiovasc Radiol $2: 149-160,1979$

38. Berger HS, Zaret BL: Nuclear Cardiology (Pt. I1) N Engl J Med 305(15):855-865. 1981

39. Berger HS. Gottschalk A, Zaret BL: Radionuclide assessment of left and right ventricular performance. Radiol Clin N Am 18:44!-466, 1980

40. Kalff V, Chan W, Rabinovitch MA, et al: Radionuclide evaluation of post extrasystolic potentiation of left ventricular function induced by atrial and ventricular stimulation. Am J Cardiol (in press)

41. Saperstein LA: Regional blood flow by fractional distribution of indicators. Am J Physiol 193:161, 1958

42. Carr EA, Gleason G. Shaw J, et al: The direct diagnosis of myocardial infarction by photoscanning after administration of Cesium-131. Am Heart J 68:627, 1964

43. Carr EA, Beierwaltes WH, Wegst AV, et al: Myocardial scanning with Rubidium-86. J Nucl Med 3:76-82, 1962

44. Cohen A, Zaleski EJ, Baleiron H, et al: Measurement of coronary blood flow using 84-Rubidium and coincidence counting methods. Am J Cardiol 19:556-562, 1967

45. Hurley PJ, Cooper M, Reba RC, et al: 43-Kcl: A new radiopharmaceutical for imaging the heart. J Nucl Med $12: 516,1971$

46. Kawsna M, Krizek H, Porter J, et al: Use of 199-TL as a potassium analog in scanning. J Nucl Med 11:333, 1970

47. Lebowitz E, Greene MW, Fairchild R, et al: Thallium-201 for medical use. I. J Nucl Med 16:151, 1975 
48. Narhara KA, Hamilton GW, Williams DL, et al: Myocardial imaging with thallium-201. An experimental model for analysis of the true myocardial and background image components. J Nucl Med 18:781, 1977

49. Goris M, Daspit SG, McLaughlin P, et al: Interpolative background subtraction. J Nucl Med 17:744-747, 1976

50. Meade RC, Virinderjit SB, Horgan JD, et al: Quantitative methods in the evaluation of thallium 201 myocardial perfusion imaging. J Nucl Med 19:1175, 1978

51. Burow RD, Pond M, Scbafer AW, et al: "Circumferential Profiles": A new method for computer analysis of thallium-201 myocardial perfusion images. J Nucl Med 20:771, 1979

52. Garcia E, Maddahi J, Berman D, et al: Space/time quantitation of thallium-201 myocardial scintigraphy. J Nucl Med 22:309,1981

53. Watson DD, Belle GA, Berger SC, et al: Notes on the quantification of sequential T1-201 images. Software 6:4-5, 10,1979

54. Watson DD, Campbell NP, Read EK, et al: Spatial and temporal quantification of plane thallium myocardial images. J Nucl Med 22:577, 1981

55. Kuhl DE, Edwards RQ: Image separation radioisotope scanning. Radiology 80:653-662, 1963

56. Radon J: On the determination of functions from their integrals along certain manifolds. Ber Verh Sachs Akad Wiss 69:262-277, 1917

57. Bracewell $R N$ : Strip integration in radio astronomy. Austr J Phys 9:198, 1956

58. DeRosier DJ, Klug A: Reconstruction of three-dimensional structures from electron micrographs. Nature 217:130-134, 1968

59. Cormack AM: Representation of a function by its line integrals with some radiological applications. Appl Phys 34:2722-2727, 1963

60. Cormack AM: Representation of a function by its line integrals with some radiological applications II. Appl Phys $35: 2908-2913,1964$

61. Housenfield GN: Computerized transverse axial scanning (tomography), Part I description of system. Br . R Radiol 46:1016-1022, 1973

62. Chesler DA: Three-dimensional activity distribution from multiple position scintigraphs. J Nucl Med 12:347, 1971

63. Kuhl DE, Edwards RQ, Ricci AR, et al: Quantitative section scanning using orthogonal tangent correction. J Nucl Med 14:196-200, 1973

64. Kay DB, Keyes JW Jr, Simon W: Radionuclide tomographic image reconstruction using Fourier transform techniques. J Nucl Med 15:981-986, 1974

65. Budinger TF: Three-dimensional imaging of the myocardium with isotopes, in Cardiovascular Imaging and Image Processing: Theory and Practice, 1975. Palos Verdes Estates, Calif. SPIE, 1976, pp 263-271

66. Brooks RA, DiChiro G: Principles of computer assisted tomography (CAT) in radiographic and radioisotopic imaging. Phys Med Biol 21:689-732, 1976

67. Budinger TF: A primer on reconstruction algorithms. Lawrence Berkeley Laboratory Report \#LBL-8212, Sept. 1978

68. Borrello JA, Clinthorne NH, Rogers WL, et al:
Oblique angle tomography-A restructuring algorithm for transaxial tomography J Nucl Med 22:471-473, 1981

69. Tamaki N, Mukai T, Ishii $Y$, et al. Clinical evaluation of thallium-201 emission myocardial tomography using a rotating gamma camera: Comparison with seven-pinhole tomography. J Nucl Med 22:849-855, 1981

70. Larson SA: Gamma camera emission tomography. Acta Radiologica [Suppl] 363, 1980

71. Maublant J, Cassagnes $J$, LeJeune JJ, et al: A comparison between conventional scintigraphy and emission tomography with thallium-201 in the detection of myocardial infarction. J Nucl Med 23:204-208, 1982

72. Gottschalk SC, Smith KA, Wake RH: Tomographic reconstruction with rotating slant hole and seven pinhole collimators on an Anger gamma camera, in Paras P, Eikman, E (eds): Emission Computed Tomography: Single Photon Approach. FDA No. 81-177, Rockville, MD, 1981, pp 105-128

73. Ratib O, lIenze E, IIoffman E, et al: Performance of the rotating slant-hole collimator for the detection of myocardial perfusion abnormalities. J Nucl Med 23:34-41, 1982

74. Nalcioglu O, Morton ME, Milne N: Computerized longitudinal tomography with a bilateral collimator. IEEE Trans Nucl Sci NS-27:430, 1980

75. Chang W, Lin SL, Henkin RE: Multisegmental collimator tomography: Comparison of the system performance of the seven pinhole and quadrant slant hole collimators, in Paras P, Eikman, E (eds): Emission Computed Tomography: Single Photon Approach. FDA No. 81-177, Rockville, 1981, pp 65-81

76. Henkin RE, Chang W, Salo BC, et al: Gamma camera tomography with multisegmental collimators: Applications to ${ }^{201} \mathrm{Tl}$ and gated blood pool imaging, in Paras P, Eikman E (eds): Emission Computed Tomography: Single Photon Approach. FDA No. 81-177, Rockville, 1981, pp 82-104

77. Chang W. Lin SL, Henkin RE: A new collimator for cardiac tomography: The quadrant slant hole collimator (QSH). J Nucl Med (Accepted for publication) 1980 (Abstr)

78. Vogel RA, Kirch DL, LeFree MT, et al: A new method of multiplanar emission tomography using a seven pinhole collimator and an Anger scintillation camera. J Nucl Med 19:648, 1978

79. Kirch DL, Vogel RA, LeFree MT, et al: An Anger camera/computer system for myocardial perfusion tomography using a seven pinhole collimator. IEEE Trans Nucl Sci NS-27:412, 1980

80. Williams DL, Ritchie JL, Hays GD, et al: In vivo simulations of thallium-201 myocardial scintigraphy by seven pinhole emission tomography. J Nucl Med 21:821-828, 1980

81. Muehllehner G: A tomographic scintillation camera. Phys Med Biol 16:87-96, 1971

82. Tam KC, Perez-Mendez V, MacDonald B: Limited angle 3-D reconstructions from continuous and pinhole projections. IEEE Trans Nuc Sci NS-27:445, 1980

83. Chiu MY, Barrett HH, Simpson RG, et al: Threedimensional radiographic imaging with a restricted view angle. J Opt Soc Am 69:1323, 1979

84. Budinger TF: Physical attributes of single photon tomography. J Nucl Med 21:579-592, 1980

85. Koral KF, Clinthorne NH, Rogers WL, et al: Feasibil- 
ity of sharpening limited-angle tomography by including an orthogonal set of projections. Nuclear Instruments and Methods 193:223-227, 1982

86. Mueller TM, Marcul MI, Erhardt IC, ef al: Limitations of thallium-201 myocardial perfusion scintigrams. Cir culation 54:640, 1976

87. Vogel RA, Kirch DL, LeFree TM, et al: Thallium-201 myocardial perfusion scintigraphy: Results of standard and multi-pinhole tomographic techniques. Am J Cardiol 43:787-793, 1979

88. Rizi HR, Kline RC. Thrall JH, et al: Thallium-201 myocardial scintigraphy: A critical comparison of seven pinhole tomography and conventional planar imaging. J Nucl Med 22:493 499, 1981

89. Vogel R, Berman D, Pitt B, et al: A multicenter comparison of standard and seven pinhole tomographic T1-
201 scintigraphy. Results of quantitative interpretation of tomograms. J Nucl Med 21:P70

90. Berman D, Freeman M. Garcia E, et al: Clinical comparison of multiple pinhole tomograplyy with planar imaging for thallium-201 stress myocardial scintigraphy. J Nucl Med 21:P70, 1980 (Abstr)

91. Williams DL, Ritchie JL, Harp GD, et al: Preliminary characterization of the properties of a transaxial whole-body single-photon tomograph: Emphasis on future applications to cardiac imaging, in Peter Esser (ed): Functional Mapping of Organ Systems and Other Computer Topics. Society of Nuclear Medicine, 1981. New York, pp 149-166

92. Circumferential Profiles: A Computer Assisted Quantitative Approach to Improved Thallium-201 Imaging. Boston, New England Nuclear 